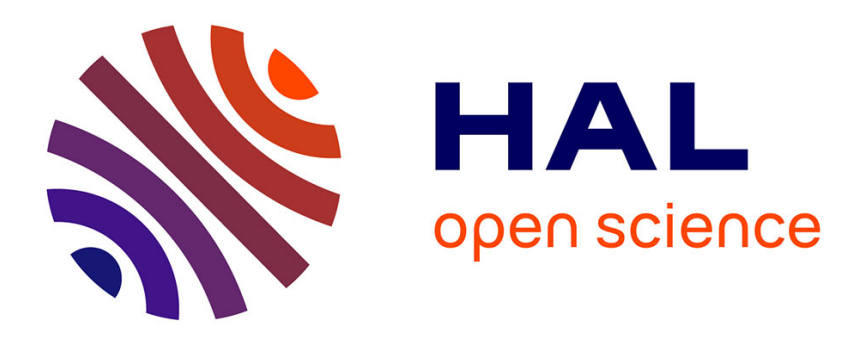

\title{
Stokes Problems in Irregular Domains with Various Boundary Conditions
}

\author{
Sylvie Monniaux, Zongwei Shen
}

\section{To cite this version:}

Sylvie Monniaux, Zongwei Shen. Stokes Problems in Irregular Domains with Various Boundary Conditions. Yoshikazu Giga, Antonin Novotny Handbook of Mathematical Analysis in Mechanics of Viscous Fluids, 59, Springer, pp.1-42, 2016, 978-3-319-10151-4. 10.1007/978-3-319-10151-4_4-1. hal-01481981

\section{HAL Id: hal-01481981 \\ https://hal.science/hal-01481981}

Submitted on 3 Mar 2017

HAL is a multi-disciplinary open access archive for the deposit and dissemination of scientific research documents, whether they are published or not. The documents may come from teaching and research institutions in France or abroad, or from public or private research centers.
L'archive ouverte pluridisciplinaire HAL, est destinée au dépôt et à la diffusion de documents scientifiques de niveau recherche, publiés ou non, émanant des établissements d'enseignement et de recherche français ou étrangers, des laboratoires publics ou privés. 


\title{
Stokes problems in irregular domains with various boundary conditions
}

\author{
Sylvie Monniaux*† Zongwei Shen ${ }^{\ddagger}$
}

\begin{abstract}
Different boundary conditions for the Navier-Stokes equations in bounded Lipschitz domains in $\mathbb{R}^{3}$, such as Dirichlet, Neumann, Hodge or Robin boundary conditions are presented here. The situation is a little different from the case of smooth domains. The analysis of the problem involves a good comprehension of the behaviour near the boundary. The linear Stokes operator associated to the various boundary conditions is first studied. Then a classical fixed point theorem is used to show how the properties of the operator lead to local solutions or global solutions for small initial data.
\end{abstract}

\section{Introduction}

The aim of this chapter is to describe how to find solutions of the Navier-Stokes equations

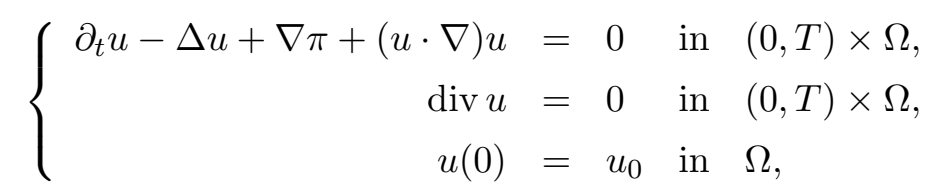

in a bounded Lipschitz domain $\Omega \subset \mathbb{R}^{3}$, and a time interval $(0, T)(T \leq \infty)$, for initial data $u_{0}$ in a critical space, with one of the following boundary conditions on $\partial \Omega$ :

1. Dirichlet boundary conditions:

$$
u=0,
$$

also called "no-slip" boundary conditions, which can be also decomposed as a non penetration condition $\nu \cdot u=0$ and a tangential part $\nu \times u=0$ which model the fact that the fluid does not slip at the boundary; this is commonly used for a boundary between a fluid and a rigid surface;

2. Neumann boundary conditions:

$$
\left[\lambda(\nabla u)+(\nabla u)^{\top}\right] \nu-\pi \nu=0, \quad \lambda \in(-1,1],
$$

which can be rewritten as $T_{\lambda}(u, \pi) \nu=0$ where $T_{\lambda}(u, \pi):=\lambda(\nabla u)+(\nabla u)^{\top}-\pi \mathrm{Id}$; if $\lambda=0$, (Nbc) becomes $\partial_{\nu} u=\pi \nu$; if $\lambda=1, T_{1}(u, \pi)$ is the Cauchy's stress tensor

\footnotetext{
*Aix-Marseille Université, CNRS, Centrale Marseille, I2M, UMR 7373, 13453 Marseille, France - email: sylvie.monniaux@univ-amu.fr

${ }^{\dagger}$ ANR HAB, Labex Archimède

${ }^{\ddagger}$ University of Kentucky, 719 Patterson Office Tower, Lexington KY 40506-0027 - email: zshen2@uky.edu
} 
so that $(\mathrm{Nbc})$ can be viewed, for instance, as an absence of stress on the interface separating two media in the case of a free boundary; ( $\mathrm{Nbc}$ ) can be decomposed into its normal and tangential parts and can be rewritten in the following form

$$
(1+\lambda) \nu \cdot \partial_{\nu} u=\pi, \quad\left[\left(\lambda(\nabla u)+(\nabla u)^{\top}\right) \nu\right]_{\tan }=0 ;
$$

3. Hodge boundary conditions:

$$
\nu \cdot u=0, \quad \nu \times \operatorname{curl} u=0,
$$

also called "absolute" boundary conditions (see [49, Section 9] or "perfect wall" condition (see [1]); they have been studied in, e.g., [4] and [23]; they are related to the more traditionally used "Navier's slip" boundary condition

$$
\left.\nu \cdot u=0, \quad\left[(\nabla u)^{\top}+\nabla u\right) \nu\right]_{\tan }=0,
$$

see discussion below (see also a detailed discussion in [34, Section 2]);

4. Robin boundary conditions:

$$
\nu \cdot u=0, \quad \nu \times \operatorname{curl} u=\alpha u, \quad \alpha>0
$$

since $\nu \cdot u=0, u$ is a tangential vector field at the boundary, so it make sense to compare it to the tangential part of the vorticity: it describes the fact that the fluid slips with a friction proportional to the vorticity. Remark that $(\mathrm{Hbc})$ is recovered if $\alpha=0$ and (Dbc) if $\alpha=\infty$.

In the boundary conditions above, $\nu(x)$ denotes the unit exterior normal vector at a point $x \in \partial \Omega$ (defined almost everywhere when $\partial \Omega$ is a Lipschitz boundary).

As explained in [34, Section 2 and Section 6], the Hodge boundary conditions (Hbc) are close to the Navier's slip boundary conditions (0.2). Indeed, if $\Omega$ is assumed to be smooth enough, say of class $\mathscr{C}^{2}$, under the condition $\nu \cdot u=0$, the following holds:

$$
\left.\left[(\nabla u)^{\top}+\nabla u\right) \nu\right]_{\tan }=-\nu \times \operatorname{curl} u+2 \mathcal{W} u
$$

where $\mathcal{W}$ is the Weingarten map (also called the shape operator, see [43, Chapter 5]) on $\partial \Omega$ acting on tangential fields (see also [17, Section 3]). In particular, the term $\mathcal{W} u$ is a zero-order term, depending linearly on the velocity field $u$, and is equal to 0 on flat portions of the boundary.

The strategy in this chapter to solve the Navier-Stokes equations with one of the boundary conditions described above is to find a functional setting in which the Fujita-Kato scheme applies, such as in their fundamental paper [20]. In all situations, the idea is to study the linear problem to prove enough regularizing properties of the Stokes semigroup so that the nonlinear problem can be treated via a fixed point method. For the last two types of boundary conditions $(\mathrm{Hbc})$ and $(\mathrm{Rbc})$, the Navier-Stokes system is rewritten as follows:

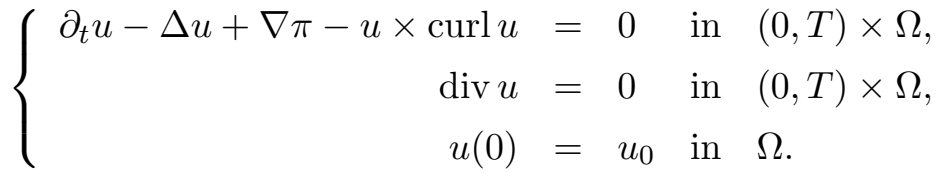


This is motivated by the form of the boundary conditions and the fact that, for a smooth enough vector field $u$,

$$
(u \cdot \nabla) u=\frac{1}{2} \nabla|u|^{2}-u \times \operatorname{curl} u,
$$

so that (NS) becomes (NS') with the pressure $\pi$ replaced by the so-called dynamical pressure $\pi+\frac{1}{2}|u|^{2}$ (see, e.g. [23] or [4]).

In this chapter, $\Omega \subset \mathbb{R}^{3}$ is a bounded, simply connected, Lipschitz domain. The chapter is organized as follows. In Section 1, the Dirichlet-Stokes operator is defined in the $L^{2}$ setting, and then in the $L^{p}$ theory. Existence of a local solution of the system $\{(\mathrm{NS}),(\mathrm{Dbc})\}$ for initial values in a critical space in the $L^{2}$-Stokes scale is then shown. In Section 2, the previous proofs are adapted in the case of Neumann boundary conditions, i.e., for the system $\{(\mathrm{NS}),(\mathrm{Nbc})\}$. In Section 3, the system $\left\{\left(\mathrm{NS}^{\prime}\right),(\mathrm{Hbc})\right\}$ is studied for initial conditions in the critical space $\left\{u \in L^{3}\left(\Omega ; \mathbb{R}^{3}\right) ; \operatorname{div} u=0\right.$ in $\Omega, \nu \cdot u=0$ on $\left.\partial \Omega\right\}$ whereas in Section 4 , the system $\left\{\left(\mathrm{NS}^{\prime}\right),(\mathrm{Rbc})\right\}$ is considered in a $\mathscr{C}^{1}$ domain.

\section{Dirichlet boundary conditions}

For a more complete exposition of the results in this section, as well as an extension to more general domains, the reader can refer to [39], [33] and [48]. The case where $\Omega$ is smooth was solved by Fujita and Kato in [20]. In [15], the case of bounded Lipschitz domains $\Omega$ was studied for initial data not in a critical space.

\subsection{The linear Dirichlet-Stokes operator}

\subsubsection{The $L^{2}$ theory}

The following remark about $L^{2}$ vector fields on $\Omega$ will be used throughout this chapter.

Remark 1.1. For $\Omega \subset \mathbb{R}^{3}$ a bounded Lipschitz domain, let $u \in L^{2}\left(\Omega ; \mathbb{R}^{3}\right)$ such that $\operatorname{div} u \in L^{2}(\Omega ; \mathbb{R})$. Then $\nu \cdot u$ can be defined on $\partial \Omega$ in the following weak sense in $H^{-\frac{1}{2}}(\partial \Omega ; \mathbb{R})$ : for $\phi \in H^{1}(\Omega ; \mathbb{R})$,

$$
\langle u, \nabla \phi\rangle_{\Omega}+\langle\operatorname{div} u, \phi\rangle_{\Omega}=\langle\nu \cdot u, \varphi\rangle_{\partial \Omega}
$$

where $\varphi=\operatorname{Tr}_{\mid \partial \Omega} \phi$, the right hand-side of (1.1) depends only on $\varphi$ on $\partial \Omega$ and not on the choice of $\phi$, its extension to $\Omega$. The notation $\langle\cdot, \cdot\rangle_{E}$ stands for the $L^{2}$-scalar product on $E$.

The following Hodge decomposition holds on vector fields: $L^{2}\left(\Omega ; \mathbb{R}^{3}\right)$ is equal to the orthogonal direct sum $H_{D} \stackrel{\perp}{\oplus} G$ where

$$
H_{D}=\left\{u \in L^{2}\left(\Omega ; \mathbb{R}^{3}\right) ; \operatorname{div} u=0 \text { in } \Omega, \nu \cdot u=0 \text { on } \partial \Omega\right\}
$$

and $G=\nabla H^{1}(\Omega ; \mathbb{R})$. This follows from the following theorem due to Georges de Rham [12, Chap.IV §22, Theorem 17']; see also [51, Chap.I§1.4, Proposition 1.1].

Theorem 1.2 (de Rham). Let $T$ be a distribution in $\mathscr{C}_{c}^{\infty}\left(\Omega ; \mathbb{R}^{3}\right)^{\prime}$ such that $\langle T, \phi\rangle=0$ for all $\phi \in \mathscr{C}_{c}^{\infty}\left(\Omega ; \mathbb{R}^{3}\right)$ with $\operatorname{div} \phi=0$ in $\Omega$. Then there exists a distribution $S \in \mathscr{C}_{c}^{\infty}(\Omega ; \mathbb{R})^{\prime}$ such that $T=\nabla S$. Conversely, if $T=\nabla S$ with $S \in \mathscr{C}_{c}^{\infty}(\Omega ; \mathbb{R})^{\prime}$, then $\langle T, \phi\rangle=0$ for all $\phi \in \mathscr{C}_{c}^{\infty}\left(\Omega ; \mathbb{R}^{3}\right)$ with $\operatorname{div} \phi=0$ in $\Omega$.

Remark 1.3. In the case of a bounded Lipschitz domain $\Omega \subset \mathbb{R}^{3}$, the space $H_{D}$ coincides with the closure in $L^{2}\left(\Omega ; \mathbb{R}^{3}\right)$ of the space of vector fields $u \in \mathscr{C}_{c}^{\infty}\left(\Omega ; \mathbb{R}^{3}\right)$ with $\operatorname{div} u=0$ in $\Omega$. 
Denote by $J: H_{D} \hookrightarrow L^{2}\left(\Omega ; \mathbb{R}^{3}\right)$ the canonical embedding and $\mathbb{P}: L^{2}\left(\Omega ; \mathbb{R}^{3}\right) \rightarrow H_{D}$ the orthogonal projection, called either Leray or Helmholtz projection. It is clear that $\mathbb{P} J=$ $\operatorname{Id}_{H_{D}}$. Define now the space $V_{D}=H_{0}^{1}\left(\Omega ; \mathbb{R}^{3}\right) \cap H_{D}$ : it is a closed subspace of $H_{0}^{1}\left(\Omega ; \mathbb{R}^{3}\right)$. The embedding $J$ restricted to $V_{D}$ maps $V_{D}$ to $H_{0}^{1}\left(\Omega ; \mathbb{R}^{3}\right)$ : denote it by $J_{0}: V_{D} \hookrightarrow H_{0}^{1}\left(\Omega ; \mathbb{R}^{3}\right)$. Its adjoint $J_{0}^{\prime}=\mathbb{P}_{1}: H^{-1}\left(\Omega ; \mathbb{R}^{3}\right) \rightarrow V_{D}^{\prime}$ is then an extension of the orthogonal projection $\mathbb{P}$. The space $H_{D}$ is endowed with the norm $u \mapsto\|u\|_{2}$ and $V_{D}$ with the norm $u \mapsto\|\nabla u\|_{2}$.

The definition of the Dirichlet-Stokes operator then follows.

Definition 1.4. The Dirichlet-Stokes operator is defined as being the associated operator of the bilinear form

$$
a: V_{D} \times V_{D} \rightarrow \mathbb{R}, \quad a(u, v)=\sum_{i=1}^{3}\left\langle\partial_{i} J_{0} u, \partial_{i} J_{0} v\right\rangle .
$$

Proposition 1.5. The Dirichlet-Stokes operator $A_{D}$ is the part in $H_{D}$ of the bounded operator $A_{0, D}: V_{D} \rightarrow V_{D}^{\prime}$ defined by $A_{0, D} u: V_{d} \rightarrow \mathbb{R},\left(A_{0, D} u\right)(v)=a(u, v)$, and satisfies

$$
\begin{gathered}
\mathrm{D}\left(A_{D}\right)=\left\{u \in V_{D} ; \mathbb{P}_{1}\left(-\Delta_{D}^{\Omega}\right) J_{0} u \in H_{D}\right\}, \\
A_{D} u=\mathbb{P}_{1}\left(-\Delta_{D}^{\Omega}\right) J_{0} u \quad u \in \mathrm{D}\left(A_{D}\right),
\end{gathered}
$$

where $\Delta_{D}^{\Omega}$ denotes the weak vector-valued Dirichlet-Laplacian in $L^{2}\left(\Omega ; \mathbb{R}^{3}\right)$. The operator $A_{D}$ is self-adjoint, invertible, $-A_{D}$ generates an analytic semigroup of contractions on $H_{D}$, $\mathrm{D}\left(A_{D}^{\frac{1}{2}}\right)=V_{D}$ and for all $u \in \mathrm{D}\left(A_{D}\right)$, there exists $\pi \in L^{2}(\Omega ; \mathbb{R})$ such that

$$
J A_{D} u=-\Delta J_{0} u+\nabla \pi
$$

and $\mathrm{D}\left(A_{D}\right)$ admits the following description

$$
\mathrm{D}\left(A_{D}\right)=\left\{u \in V_{D} ; \exists \pi \in L^{2}(\Omega ; \mathbb{R}):-\Delta J_{0} u+\nabla \pi \in H_{D}\right\} .
$$

Proof. By definition, for $u \in \mathrm{D}\left(A_{D}\right)$ and for all $v \in V_{D}$,

$$
\begin{aligned}
\left\langle A_{D} u, v\right\rangle & =a(u, v)=\sum_{j=1}^{n}\left\langle\partial_{j} J_{0} u, \partial_{j} J_{0} v\right\rangle \\
& =-\sum_{j=1}^{n} H^{-1}\left\langle\partial_{j}^{2} J_{0} u, J_{0} v\right\rangle_{H_{0}^{1}}=H^{-1}\left\langle(-\Delta) J_{0} u, J_{0} v\right\rangle_{H_{0}^{1}} \\
& ={ }_{V_{D}^{\prime}}\left\langle\mathbb{P}_{1}(-\Delta) J_{0} u, v\right\rangle_{V_{D}} .
\end{aligned}
$$

The third equality comes from the definition of weak derivatives in $L^{2}$, the fourth equality comes from the fact that $\sum_{j=1}^{n} \partial_{j}^{2}=\Delta$. The last equality is due to the fact that $J_{0}^{\prime}=\mathbb{P}_{1}$. Therefore, $A_{D} u$ and $\mathbb{P}_{1}(-\Delta) J_{0} u$ are two linear forms which coincide on $V_{D}$, they are then equal, which proves that $A_{0, D}=\mathbb{P}_{1}(-\Delta) J_{0}: V_{D} \rightarrow V_{D}^{\prime}$. Moreover, the fact that $u \in D\left(A_{D}\right)$ implies that $A_{D} u$ is a linear form on $H_{D}$, so that the linear form $\mathbb{P}_{1}(-\Delta) J_{0} u$, originally defined on $V_{D}$, extends to a linear form on $H_{D}$ (since $V_{D}$ is dense in $H_{D}$ by de Rham's theorem). The fact that $A_{D}$ is self-adjoint and $-A_{D}$ generates an analytic semigroup of contractions comes from the properties of the form $a$ : $a$ is bilinear, symmetric, sectorial of angle 0 , coercive on $V_{D} \times V_{D}$. The property that $\mathrm{D}\left(A_{D}^{\frac{1}{2}}\right)=V_{D}$ is due to the fact that $A_{D}$ is self-adjoint, applying a result by J.L. Lions [28, Théorème 5.3]. 
To prove the last assertions of this proposition, let $u \in \mathrm{D}\left(A_{D}\right)$. Then $A_{D} u \in H_{D}$ and $\mathbb{P}_{1} J\left(A_{D} u\right)=\mathbb{P} J\left(A_{D} u\right)=u$. Moreover, if $u \in \mathrm{D}\left(A_{D}\right), u$ belongs, in particular, to $V_{D}$. Therefore, $J_{0} u \in H_{0}^{1}\left(\Omega ; \mathbb{R}^{3}\right)$ and $(-\Delta) J_{0} u \in H^{-1}\left(\Omega ; \mathbb{R}^{3}\right)$. The following identities take place in $V_{D}^{\prime}$,

$$
\mathbb{P}_{1}\left(J\left(A_{D} u\right)-(-\Delta) J_{0} u\right)=\mathbb{P}_{1} J\left(A_{D} u\right)-\mathbb{P}_{1}(-\Delta) J_{0} u=A_{D} u-A_{D} u=0 .
$$

By de Rham's theorem, this implies that there exists $p \in \mathscr{C}_{c}^{\infty}(\Omega ; \mathbb{R})^{\prime}$ such that $J\left(A_{D} u\right)-$ $(-\Delta) \tilde{J} u=\nabla p: \nabla p \in H^{-1}\left(\Omega ; \mathbb{R}^{3}\right)$, which implies that $p \in L^{2}(\Omega ; \mathbb{R})$.

The relations between the spaces and the operators described above are summarized in the following commutative diagram:

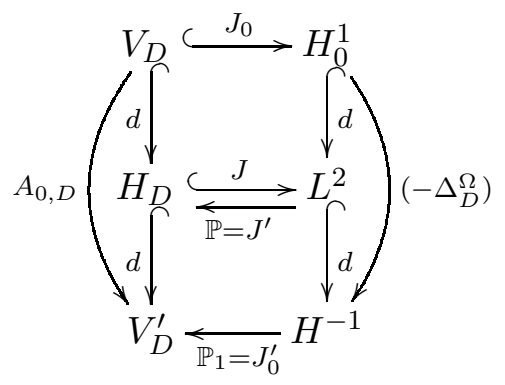

In the case of a bounded Lipschitz domain $\Omega \subset \mathbb{R}^{3}$, the following property of $\mathrm{D}\left(A_{D}^{\frac{3}{4}}\right)$ also holds; see [33, Corollary 5.5].

Proposition 1.6. The domain of $A_{D}^{\frac{3}{4}}$ is continuously embedded into $W_{0}^{1,3}\left(\Omega ; \mathbb{R}^{3}\right)$.

It has been proved by R. Brown and Z. Shen [7] that the domain of $A_{D}$ is embedded into $W_{0}^{1, p}\left(\Omega ; \mathbb{R}^{3}\right) \cap W^{\frac{3}{2}, 2}\left(\Omega, \mathbb{R}^{3}\right)$ for some $p>3$. The proof Proposition 1.6 uses the well posedness result for the Poisson problem of the Stokes system [16, Theorem 5.6], similar to the corresponding result proved in [25] for the Laplacian.

\subsubsection{The $L^{p}$ theory}

P. Deuring provided in [14] an example of a domain with one conical singularity such that the Dirichlet-Stokes semigroup does not extend to an analytic semigroup in $L^{p}$ for $p$ large, away from 2. M.E. Taylor in [50], however, conjectured that this should be true for $p$ in an interval containing $\left[\frac{3}{2}, 3\right]$, which was indeed proved 12 years later by the second author in [48].

Let $\mathscr{C}_{c, \sigma}^{\infty}(\Omega)$ denote the space of vector fields $u \in \mathscr{C}_{c}^{\infty}\left(\Omega ; \mathbb{R}^{3}\right)$ with $\operatorname{div} u=0$ in $\Omega$, and

$$
L_{\sigma}^{p}(\Omega)=\text { the closure of } \mathscr{C}_{c, \sigma}^{\infty}(\Omega) \text { in } L^{p}\left(\Omega ; \mathbb{R}^{3}\right) .
$$

Note that if $\Omega$ is Lipschitz and $p=2, L_{\sigma}^{2}(\Omega)=H_{D}$. In view of Proposition 1.5, the Dirichlet-Stokes operator in the $L^{p}$ setting for $1<p<\infty$ is defined by

$$
A_{D, p}=-\Delta u+\nabla \pi
$$

with the domain

$$
\begin{aligned}
\mathrm{D}\left(A_{D, p}\right)=\left\{u \in W_{0}^{1, p}\left(\Omega ; \mathbb{R}^{3}\right) ; \operatorname{div} u=0 \text { in } \Omega\right. \text { and } \\
\left.\qquad-\Delta u+\nabla \pi \in L_{\sigma}^{p}(\Omega) \text { for some } \pi \in L^{p}(\Omega)\right\} .
\end{aligned}
$$


Since $\mathscr{C}_{c, \sigma}^{\infty}(\Omega) \subset \mathrm{D}\left(A_{D, p}\right)$, the operator $A_{D, p}$ is densely defined in $L_{\sigma}^{p}(\Omega)$ and $A_{D, p}(u)=$ $\mathbb{P}(-\Delta) u$ for $u \in \mathscr{C}_{c, \sigma}^{\infty}(\Omega)$. If $p=2, A_{D, p}$ agrees with the Dirichlet-Stokes operator $A_{D}$ defined in the previous subsection.

The following theorem was proved in [48].

Theorem 1.7. Let $\Omega$ be a bounded Lipschitz domain in $\mathbb{R}^{3}$. Then there exists $\varepsilon>0$, depending only on the Lipschitz character of $\Omega$, such that $-A_{D, p}$ generates a bounded analytic semigroup in $L_{\sigma}^{p}(\Omega)$ for $(3 / 2)-\varepsilon<p<3+\varepsilon$.

It was in fact proved in [48] that if $\Omega$ is a bounded Lipschitz domain in $\mathbb{R}^{d}, d \geq 3$, then $-A_{D, p}$ generates a bounded analytic semigroup in $L_{\sigma}^{p}(\Omega)$ for

$$
\frac{2 d}{d+1}-\varepsilon<p<\frac{2 d}{d-1}+\varepsilon
$$

where $\varepsilon>0$ depends only on $d$ and the Lipschitz character of $\Omega$. This was done by establishing the following resolvent estimate in $L^{p}$,

$$
\left\|\left(A_{D, p}+\lambda\right)^{-1} f\right\|_{L^{p}\left(\Omega ; \mathbb{C}^{d}\right)} \leq C_{p}|\lambda|^{-1}\|f\|_{L^{p}\left(\Omega ; \mathbb{C}^{d}\right)}
$$

for any $f \in \mathscr{C}_{c}^{\infty}\left(\Omega ; \mathbb{C}^{d}\right)$ with $\operatorname{div} f=0$ in $\Omega$, where $p$ satisfies (1.7),

$$
\lambda \in \Sigma_{\theta}:=\{z \in \mathbb{C}: \lambda \neq 0 \text { and }|\arg (z)|<\pi-\theta\},
$$

and $\theta \in(0, \pi / 2)$. The constant $C_{p}$ in (1.8) depends only on $d, \theta, p$, and $\Omega$. It has long been known that if $\Omega$ is a bounded $\mathscr{C}^{2}$ domain in $\mathbb{R}^{d}$, the resolvent estimate (1.8) holds for $\lambda \in \Sigma_{\theta}$ and $1<p<\infty$ (see [21]). Consequently, the operator $A_{D, p}$ generates a bounded analytic semigroup in $L^{p}$ for any $1<p<\infty$, if $\Omega$ is $\mathscr{C}^{2}$. The case of nonsmooth domains is much more delicate. As mentioned earlier, P. Deuring constructed a three-dimensional Lipschitz domain for which the $L^{p}$ resolvent estimate (1.8) fails for $p$ sufficiently large. This was somewhat unexpected. Indeed it was proved in [45] that the $L^{p}$ resolvent estimate holds for $1<p<\infty$ in bounded Lipschitz domains in $\mathbb{R}^{3}$ for any second-order elliptic systems with constant coefficients satisfying the Legendre-Hadamard conditions (the range is $\frac{2 d}{d+3}-\varepsilon<p<\frac{2 d}{d-3}+\varepsilon$ for $d \geq 4$ ). It is worth mentioning that it is not known whether the range of $p$ in Theorem 1.7 is sharp.

The approach used in [48] to the proof of (1.8) is described below. Consider the operator $T_{\lambda}$ on $L^{2}\left(\Omega ; \mathbb{C}^{d}\right)$, defined by $T_{\lambda}(f)=\lambda u$, where $\lambda \in \Sigma_{\theta}$ and $u \in H_{0}^{1}\left(\Omega ; \mathbb{C}^{d}\right)$ is the unique solution to the Stokes system

$$
\left\{\begin{array}{rlrl}
-\Delta u+\nabla \pi+\lambda u=f & & \text { in } \Omega, \\
\operatorname{div} u=0 & & \text { in } \Omega, \\
u & =0 & & \text { on } \partial \Omega .
\end{array}\right.
$$

Note that $T_{\lambda}$ is bounded on $L^{2}\left(\Omega ; \mathbb{C}^{d}\right)$ and $\left\|T_{\lambda}\right\|_{L^{2} \rightarrow L^{2}} \leq C$. To show that $T_{\lambda}$ is bounded on $L^{p}\left(\Omega ; \mathbb{C}^{d}\right)$ and $\left\|T_{\lambda}\right\|_{L^{p} \rightarrow L^{p}} \leq C$ for $2<p<\frac{2 d}{d-1}+\varepsilon$, a real variable argument is used, which may be regarded as a refined (and dual) version of the celebrated Calderón-Zygmund Lemma. According to this argument, which originated from [8] and further developed in $[46,47]$, one only needs to establish the weak reverse Hölder estimate,

$$
\left(f_{B\left(x_{0}, r\right) \cap \Omega}|u|^{p_{d}}\right)^{1 / p_{d}} \leq C\left(f_{B\left(x_{0}, 2 r\right) \cap \Omega}|u|^{2}\right)^{1 / 2}
$$


for $p_{d}=\frac{2 d}{d-1}$, whenever $u \in H_{0}^{1}\left(\Omega ; \mathbb{C}^{d}\right)$ is a (local) solution of the Stokes system

$$
\left\{\begin{aligned}
-\Delta u+\nabla \pi+\lambda u & =0 \\
\operatorname{div} u & =0
\end{aligned}\right.
$$

in $B\left(x_{0}, 3 r\right) \cap \Omega$ for some $x_{0} \in \bar{\Omega}$ and $0<r<c \operatorname{diam}(\Omega)$. The extra $\varepsilon$ in the range of $p$ is due to the self-improvement property of the weak reverse Hölder inequalities (see, e.g., [24]).

To prove the estimate (1.10), the Dirichlet problem for the Stokes system (1.11) is considered in a bounded Lipschitz domain $\Omega$ in $\mathbb{R}^{d}$, with boundary data $u=f$ on $\partial \Omega$, where $f \in L^{2}\left(\partial \Omega ; \mathbb{C}^{d}\right)$ and $\int_{\partial \Omega} f \cdot \nu=0$. The goal is to show that

$$
\left\|(u)^{*}\right\|_{L^{2}(\partial \Omega)} \leq C\|f\|_{L^{2}(\partial \Omega)},
$$

where $(u)^{*}$ denotes the nontangential maximal function of $u$ and is defined by

$$
(u)^{*}(Q):=\sup \left\{|u(x)|: x \in \Omega \text { and }|x-Q|<C_{0} \operatorname{dist}(x, \partial \Omega)\right\}
$$

for any $Q \in \partial \Omega\left(C_{0}>1\right.$ is a large fixed constant depending on $d$ and $\left.\Omega\right)$. This, together with the inequality

$$
\left(\int_{\Omega}|u|^{p_{d}}\right)^{1 / p_{d}} \leq C\left(\int_{\partial \Omega}\left|(u)^{*}\right|^{2}\right)^{1 / 2}
$$

which holds for any continuous function $u$ in $\Omega$, leads to

$$
\left(\int_{\Omega}|u|^{p_{d}}\right)^{1 / p_{d}} \leq C\left(\int_{\partial \Omega}|u|^{2}\right)^{1 / 2} .
$$

The desired estimate (1.10) follows by applying (1.13) in the domain $B\left(x_{0}, t r\right) \cap \Omega$ for $t \in(1,2)$ and then integrating the resulting inequality with respect to $t$ over $(1,2)$.

Finally, the nontangential-maximal-function estimate (1.12) is established by the method of layer potentials. The case $\lambda=0$ was studied in [11, 18], where the $L^{2}$ Dirichlet problem as well as the Neumann type boundary value problems with boundary data in $L^{2}$ for the system $-\Delta u+\nabla \pi=0$ and $\operatorname{div} u=0$ in a Lipschitz domain $\Omega$ was solved by the method of layer potentials, using the Rellich type estimates

$$
\left\|\frac{\partial u}{\partial \rho}\right\|_{L^{2}(\partial \Omega)} \approx\left\|\nabla_{\tan } u\right\|_{L^{2}(\partial \Omega)} .
$$

Here $\frac{\partial u}{\partial \rho}$ is a conormal derivative and $\nabla_{\tan } u$ denotes the tangential derivative of $u$ on $\partial \Omega$. The reader is referred to the book [26] by C. Kenig for references on related work on $L^{p}$ boundary value problems for elliptic and parabolic equations in nonsmooth domains. In an effort to solve the $L^{2}$ initial boundary value problems for the nonstationary Stokes equations $\partial_{t} u-\Delta u+\nabla \pi=0$ and $\operatorname{div} u=0$ in a Lipschitz cylinder $(0, T) \times \Omega$, the Stokes system (1.11) for $\lambda=i \tau$ with $\tau \in \mathbb{R}$ was considered by the second author in [44]. One of the key observations in [44] is that if $\lambda=i \tau$ and $\tau \in \mathbb{R}$ is large, the Rellich estimates for the system (1.11) involve two extra terms $|\tau|^{1 / 2}\|u\|_{L^{2}(\partial \Omega)}$ and $|\tau|\|u \cdot \nu\|_{H^{-1}(\partial \Omega)}$, where $H^{-1}(\partial \Omega)$ denotes the dual of $H^{1}(\partial \Omega)$. While the first term $|\tau|^{1 / 2}\|u\|_{L^{2}(\partial \Omega)}$ was expected in view of the Rellich estimates for the Helmholtz equation $-\Delta+i \tau$ in [6], the second term $|\tau|\|u \cdot \nu\|_{H^{-1}(\partial \Omega)}$ was not. Let

$$
\frac{\partial u}{\partial \rho}=\frac{\partial u}{\partial \nu}-\pi \nu .
$$


By following the general approach in [44], it was proved in [48] that if $(u, \pi)$ is a suitable solution of (1.11) in $\Omega$, then

$$
\left\|\frac{\partial u}{\partial \rho}\right\|_{L^{2}(\partial \Omega)} \approx\left\|\nabla_{\tan } u\right\|_{L^{2}(\partial \Omega)}+|\lambda|^{1 / 2}\|u\|_{L^{2}(\partial \Omega)}+|\lambda|\|u \cdot \nu\|_{H^{-1}(\partial \Omega)}
$$

holds uniformly in $\lambda$ for $\lambda \in \Sigma_{\theta}$ with $|\lambda| \geq c>0$. As in the case of Laplace's equation [52], the estimate (1.12) follows from (1.14) by the method of layer potentials. The reader is referred to [48] for the details.

\subsection{The nonlinear Dirichlet-Navier-Stokes equations}

The system $\{(\mathrm{NS}),(\mathrm{Dbc})\}$ is invariant under the scaling $u_{\lambda}(t, x)=\lambda u\left(\lambda^{2} t, \lambda x\right),\left(\lambda^{2} t, \lambda x\right) \in$ $(0, T) \times \Omega(\lambda>0)$ : if $u$ is a solution of $\{(\mathrm{NS}),(\mathrm{Dbc})\}$ in $(0, T) \times \Omega$ for the initial value $u_{0}$, then $u_{\lambda}$ is a solution of $\{(\mathrm{NS}),(\mathrm{Dbc})\}$ in $\left(0, \frac{T}{\lambda^{2}}\right) \times \frac{1}{\lambda} \Omega$ for the initial value $x \mapsto \lambda u_{0}(\lambda x)$.

The goal here is to find the so-called mild solutions of the system $\{(\mathrm{NS}),(\mathrm{Dbc})\}$ for initial values $u_{0}$ in a critical space, in the same spirit as in [20].

Lemma 1.8. The space $\mathrm{D}\left(A_{D}^{\frac{1}{4}}\right)$ is a critical space for the Navier-Stokes equations.

Proof. The space $\mathrm{D}\left(A_{D}^{\frac{1}{4}}\right)$ is invariant under the scaling $u_{\lambda}(x)=\lambda u_{0}(\lambda x)$ for $x \in \frac{1}{\lambda} \Omega, \lambda>0$. Indeed, it suffices to check that $\left\|u_{\lambda}\right\|_{2}=\lambda^{-\frac{1}{2}}\|u\|_{2}$ and $\left\|\nabla u_{\lambda}\right\|_{2}=\lambda^{\frac{1}{2}}\|\nabla u\|_{2}$ and apply the fact that $\mathrm{D}\left(A_{D}^{\frac{1}{4}}\right)$ is the interpolation space (with coefficient $\frac{1}{2}$ ) between $H_{D}$, closed subspace of $L^{2}\left(\Omega ; \mathbb{R}^{3}\right)$, and $V_{D}=\mathrm{D}\left(A_{D}^{\frac{1}{2}}\right)$, closed subspace of $H_{0}^{1}\left(\Omega ; \mathbb{R}^{3}\right)$.

For $T>0$, define the space $\mathscr{E}_{T}$ by

$$
\begin{gathered}
\mathscr{E}_{T}=\left\{u \in \mathscr{C}_{b}\left([0, T] ; \mathrm{D}\left(A_{D}^{\frac{1}{4}}\right)\right) ; u(t) \in \mathrm{D}\left(A_{D}^{\frac{3}{4}}\right), u^{\prime}(t) \in \mathrm{D}\left(A_{D}^{\frac{1}{4}}\right) \text { for all } t \in(0, T]\right. \\
\text { and } \left.\sup _{t \in(0, T)}\left\|t^{\frac{1}{2}} A_{D}^{\frac{3}{4}} u(t)\right\|_{2}+\sup _{t \in(0, T)}\left\|t A_{D}^{\frac{1}{4}} u^{\prime}(t)\right\|_{2}<\infty\right\}
\end{gathered}
$$

endowed with the norm

$$
\|u\|_{\mathscr{E}_{T}}=\sup _{t \in(0, T)}\left\|A_{D}^{\frac{1}{4}} u(t)\right\|_{2}+\sup _{t \in(0, T)}\left\|t^{\frac{1}{2}} A_{D}^{\frac{3}{4}} u(t)\right\|_{2}+\sup _{t \in(0, T)}\left\|t A_{D}^{\frac{1}{4}} u^{\prime}(t)\right\|_{2} .
$$

The fact that $\mathscr{E}_{T}$ is a Banach space is straightforward. Assume now that $u \in \mathscr{E}_{T}$, and that $\left(J_{0} u, p\right)$ (with $p \in L^{2}(\Omega ; \mathbb{R})$ ) satisfy $\{(\mathrm{NS}),(\mathrm{Dbc})\}$ in $H^{-1}\left(\Omega ; \mathbb{R}^{3}\right)$ : indeed, every term $\nabla p, \partial_{t} J_{0} u,-\Delta J_{0} u$ and $\left(J_{0} u \cdot \nabla\right) J_{0} u$ independently belong to $H^{-1}\left(\Omega ; \mathbb{R}^{3}\right)$. Apply $\mathbb{P}_{1}$ to the equations and obtain

$$
u^{\prime}(t)+A_{D} u(t)=-\mathbb{P}_{1}\left(\left(J_{0} u \cdot \nabla\right) J_{0} u\right)
$$

since $\mathbb{P}_{1} \nabla p=0$ and $\mathbb{P}_{1}(-\Delta) J_{0} u=A_{0, D} u$. The problem $\{(\mathrm{NS}),(\mathrm{Dbc})\}$ is then reduced to the abstract Cauchy problem

$$
\begin{aligned}
u^{\prime}(t)+A_{0, D} u(t) & =-\mathbb{P}_{1}\left(\left(J_{0} u \cdot \nabla\right) J_{0} u\right) \\
u(0) & =u_{0}, \quad u \in \mathscr{E}_{T},
\end{aligned}
$$

for which a mild solution is given by the Duhamel formula:

$$
u=\alpha+\phi(u, u)
$$


where $\alpha(t)=e^{-t A_{D}} u_{0}$ and

$$
\phi(u, v)(t)=\int_{0}^{t} e^{-(t-s) A_{D}}\left(-\frac{1}{2} \mathbb{P}_{1}\left(\left(J_{0} u(s) \cdot \nabla\right) J_{0} v(s)+\left(J_{0} v(s) \cdot \nabla\right) J_{0} u(s)\right)\right) \mathrm{d} s .
$$

The strategy to find $u \in \mathscr{E}_{T}$ satisfying $u=\alpha+\phi(u, u)$ is to apply a fixed point theorem. For that, $\mathscr{E}_{T}$ needs to be a "good" space for the problem, i.e., $\alpha \in \mathscr{E}_{T}$ and $\phi(u, u) \in \mathscr{E}_{T}$. The fact that $\alpha \in \mathscr{E}_{T}$ follows directly from the properties of the Stokes operator $A_{D}$ and the semigroup $\left(e^{-t A_{D}}\right)_{t \geq 0}$.

Proposition 1.9. The mapping $\phi: \mathscr{E}_{T} \times \mathscr{E}_{T} \rightarrow \mathscr{E}_{T}$ is bilinear, continuous and symmetric.

Proof. The fact that $\phi$ is bilinear and symmetric is immediate, once it is proved that it is well-defined. For $u, v \in \mathscr{E}_{T}$, let

$$
f(t)=-\frac{1}{2} \mathbb{P}_{1}\left(\left(J_{0} u(t) \cdot \nabla\right) J_{0} v(t)+\left(J_{0} v(t) \cdot \nabla\right) J_{0} u(t)\right), \quad t \in(0, T) .
$$

By the definition of $\mathscr{E}_{T}$ and Sobolev embeddings, it is easy to see that

$$
\left(J_{0} u(t) \cdot \nabla\right) J_{0} v(t)+\left(J_{0} v(t) \cdot \nabla\right) J_{0} u(t) \in L^{2}\left(\Omega ; \mathbb{R}^{3}\right)
$$

and

$$
\left\|\left(J_{0} u(t) \cdot \nabla\right) J_{0} v(t)+\left(J_{0} v(t) \cdot \nabla\right) J_{0} u(t)\right\|_{2} \leq C t^{-\frac{3}{4}}\|u\|_{\mathscr{E}_{T}}\|v\|_{\mathscr{E}_{T}}
$$

where $C$ is a constant independent from $t$, which gives the following estimate

$$
\|f(t)\|_{2} \leq C t^{-\frac{3}{4}}\|u\|_{\mathscr{E}_{T}}\|v\|_{\mathscr{E}_{T}}
$$

Therefore,

$$
\begin{aligned}
\left\|A_{D}^{\frac{1}{4}} \phi(u, v)(t)\right\|_{2} & \leq \int_{0}^{t}\left\|A_{D}^{\frac{1}{4}} e^{-(t-s) A_{D}}\right\|_{\mathscr{L}\left(H_{D}\right)} C s^{-\frac{3}{4}}\|u\|_{\mathscr{E}_{T}}\|v\|_{\mathscr{E}_{T}} \mathrm{~d} s \\
& \leq C\left(\int_{0}^{t}(t-s)^{-\frac{1}{4}} s^{-\frac{3}{4}} \mathrm{~d} s\right)\|u\|_{\mathscr{E}_{T}}\|v\|_{\mathscr{E}_{T}},
\end{aligned}
$$

and since $\int_{0}^{t}(t-s)^{-\frac{1}{4}} s^{-\frac{3}{4}} \mathrm{~d} s=\int_{0}^{1}(1-s)^{-\frac{1}{4}} s^{-\frac{3}{4}} \mathrm{~d} s$, the following estimate is finally obtained:

$$
\left\|A_{D}^{\frac{1}{4}} \phi(u, v)(t)\right\|_{2} \leq C\|u\|_{\mathscr{E}_{T}}\|v\|_{\mathscr{E}_{T}} .
$$

The proof of the continuity of $t \mapsto A_{D}^{\frac{1}{4}} \phi(u, v)(t)$ on $H_{D}$ is straightforward once the estimate (1.20) is established. The proof of the fact that

$$
\left\|\sqrt{t} A_{D}^{\frac{3}{4}} \phi(u, v)(t)\right\|_{2} \leq C\|u\|_{\mathscr{E}_{T}}\|v\|_{\mathscr{E}_{T}}
$$

is proved the same way, replacing $A_{D}^{\frac{1}{4}}$ by $A_{D}^{\frac{3}{4}}$ and using the fact that

$$
\left\|A_{D}^{\frac{3}{4}} e^{-(t-s) A_{D}}\right\|_{\mathscr{L}\left(H_{D}\right)} \leq C(t-s)^{-\frac{3}{4}}
$$

and

$$
\int_{0}^{t}(t-s)^{-\frac{3}{4}} s^{-\frac{3}{4}} \mathrm{~d} s=t^{-\frac{1}{2}} \int_{0}^{1}(1-s)^{-\frac{3}{4}} s^{-\frac{3}{4}} \mathrm{~d} s
$$


It remains to prove the estimate on the derivative with respect to $t$ of $\phi(u, v)$. Rewrite $f$ as defined in (1.18) as follows:

$$
f(s)=-\frac{1}{2} \mathbb{P}_{1} \nabla \cdot\left(J_{0} u(s) \otimes J_{0} v(s)+J_{0} v(s) \otimes J_{0} u(s)\right)
$$

where $u \otimes v$ denotes the matrix $\left(u_{i} v_{j}\right)_{1 \leq i, j \leq 3}$ and the differential operator $\nabla \cdot$ acts on matrices $M=\left(m_{i, j}\right)_{1 \leq i, j \leq 3}$ the following way:

$$
\nabla \cdot M=\left(\sum_{i=1}^{3} \partial_{i} m_{i, j}\right)_{1 \leq j \leq 3}
$$

For $u, v \in \mathscr{E}_{T}$ and $s \in(0, T)$,

$$
\begin{aligned}
f^{\prime}(s)= & -\frac{1}{2} \mathbb{P}_{1} \nabla \cdot\left(J u^{\prime}(s) \otimes J_{0} v(s)+J_{0} u(s) \otimes J v^{\prime}(s)\right. \\
& \left.+J v^{\prime}(s) \otimes J_{0} u(s)+J_{0} v(s) \otimes J u^{\prime}(s)\right)
\end{aligned}
$$

For all $s \in(0, T)$

$$
\begin{aligned}
s^{\frac{5}{4}}\left\|J u^{\prime}(s) \otimes J_{0} v(s)\right\|_{2} & \leq\left\|s J u^{\prime}(s)\right\|_{3}\left\|s^{\frac{1}{4}} J_{0} v(s)\right\|_{6} \\
& \leq\left\|s A_{D}^{\frac{1}{4}} u^{\prime}(s)\right\|_{2}\left\|s^{\frac{1}{4}} A_{D}^{\frac{1}{2}} v(s)\right\|_{2} \\
& \leq\|u\|_{\mathscr{E}_{T}}\|v\|_{\mathscr{E}_{T}},
\end{aligned}
$$

where the first inequality comes from the fact that $L^{3} \cdot L^{6} \hookrightarrow L^{2}$, the second comes from the Sobolev embeddings $\mathrm{D}\left(A_{D}^{\frac{1}{4}}\right) \hookrightarrow L^{3}\left(\Omega ; \mathbb{R}^{3}\right)$ and $\mathrm{D}\left(A_{D}^{\frac{1}{2}}\right) \hookrightarrow L^{6}\left(\Omega ; \mathbb{R}^{3}\right)$ and the third inequality follows directly from the definition of the space $\mathscr{E}_{T}$. Of course the same occurs for the other three terms $J_{0} u(s) \otimes J v^{\prime}(s), J v^{\prime}(s) \otimes J_{0} u(s)$ and $J_{0} v(s) \otimes J u^{\prime}(s)$. Therefore, since $A_{D}^{-\frac{1}{2}}$ maps $V_{d}^{\prime}$ to $H_{D}$,

$$
\sup _{0<s<T}\left\|s^{\frac{5}{4}} A_{D}^{-\frac{1}{2}} f^{\prime}(s)\right\|_{2} \leq c\|u\|_{\mathscr{E}_{T}}\|v\|_{\mathscr{E}_{T}} .
$$

It is straightforward that

$$
\phi(u, v)(t)=\int_{0}^{\frac{t}{2}} e^{-s A_{D}} f(t-s) d s+\int_{0}^{\frac{t}{2}} e^{-(t-s) A_{D}} f(s) \mathrm{d} s \quad t \in(0, T),
$$

and therefore

$$
\begin{aligned}
\phi(u, v)^{\prime}(t)= & e^{-\frac{t}{2} A_{D}} f\left(\frac{t}{2}\right)+\int_{0}^{\frac{t}{2}} A_{D}^{\frac{1}{2}} e^{-s A_{D}} A_{0, D}^{-\frac{1}{2}} f^{\prime}(t-s) \mathrm{d} s \\
& +\int_{0}^{\frac{t}{2}}-A_{D} e^{-(t-s) A_{D}} f(s) \mathrm{d} s,
\end{aligned}
$$

which yields

$$
\begin{aligned}
\left\|A_{D}^{\frac{1}{4}} \phi(u, v)^{\prime}(t)\right\|_{2} \leq & \frac{c}{t^{\frac{1}{4}}}\left\|f\left(\frac{t}{2}\right)\right\|_{2}+c\left(\int_{0}^{\frac{t}{2}} \frac{1}{s^{\frac{3}{4}}} \frac{1}{(t-s)^{\frac{5}{4}}} \mathrm{~d} s\right)\|u\|_{\mathscr{E}_{T}}\|v\|_{\mathscr{E}_{T}} \\
& +c\left(\int_{0}^{\frac{t}{2}} \frac{1}{(t-s)^{\frac{5}{4}}} \frac{1}{s^{\frac{3}{4}}} \mathrm{~d} s\right)\|u\|_{\mathscr{E}_{T}}\|v\|_{\mathscr{E}_{T}} \\
\leq & \frac{c}{t}\left(1+\int_{0}^{\frac{1}{2}} \frac{\mathrm{d} \sigma}{(1-\sigma)^{\frac{5}{4}} \sigma^{\frac{3}{4}}}\right)\|u\|_{\mathscr{E}_{T}}\|v\|_{\mathscr{E}_{T}},
\end{aligned}
$$


where the estimates (1.19), (1.23), and the fact that $-A_{D}$ generates a bounded analytic semigroup (so that $\left\|A_{D}^{\alpha} e^{-t A_{D}}\right\|_{\mathscr{L}\left(H_{D}\right)} \leq C t^{-\alpha}$ ) were used. This last inequality together with (1.20) and (1.21) ensure that $\phi(u, v) \in \mathscr{E}_{T}$ whenever $u, v \in \mathscr{E}_{T}$.

This section is concludedby applying Picard's fixed point theorem (see, e.g., [27, Theorem 13.2] or [40, Theorem A.1]) to obtain the following existence result for the system $\{(\mathrm{NS}),(\mathrm{Dbc})\}$.

Theorem 1.10 (Existence). Let $\Omega \subset \mathbb{R}^{3}$ be a bounded Lipschitz domain and let $u_{0} \in$ $\mathrm{D}\left(A_{D}^{\frac{1}{4}}\right)$. Let $\alpha$ and $\phi$ be defined as above.

(i) If $\left\|A_{D}^{\frac{1}{4}} u_{0}\right\|_{2}$ is small enough, then there exists a unique $u \in \mathscr{E}_{\infty}$ solution of $u=$ $\alpha+\phi(u, u)$.

(ii) For all $u_{0} \in \mathrm{D}\left(A_{D}^{\frac{1}{4}}\right)$, there exists $T>0$ and a unique $u \in \mathscr{E}_{T}$ solution of $u=\alpha+\phi(u, u)$.

Uniqueness in the larger space $\mathscr{C}_{b}\left([0, T) ; \mathrm{D}\left(A_{D}^{\frac{1}{4}}\right)\right)$ can be obtained, applying [38, Theorem 1.1]. The argument there is somewhat stronger though, since uniqueness in $\mathscr{C}_{b}\left([0, T) ; L^{3}\right)$ is proved, using a maximal regularity result by Z. Shen [44, Theorem 5.1.2].

Theorem 1.11 (Uniqueness). Let $u, v \in \mathscr{C}_{b}\left([0, T) ; \mathrm{D}\left(A_{D}^{\frac{1}{4}}\right)\right)$ both be mild solutions of the system $\{(\mathrm{NS}),(\mathrm{Dbc})\}$, i.e., they both satisfy (1.16). Then $u=v$ on $[0, T)$.

Before proving this theorem, the following lemma is shown, similar to [37, Proposition 2].

Lemma 1.12. Let $p \in(1, \infty)$ and $\tau \in(0, T]: \phi$ defined by (1.17) maps $L^{p}\left(0, \tau ; \mathrm{D}\left(A_{D}^{\frac{1}{4}}\right)\right) \times$ $L^{\infty}\left(0, \tau ; \mathrm{D}\left(A_{D}^{\frac{1}{4}}\right)\right)$ to $L^{p}\left(0, \tau ; \mathrm{D}\left(A_{D}^{\frac{1}{4}}\right)\right)$. Moreover, there exists a constant $C_{p}>0$ independent of $\tau$ such that

$$
\|\phi(u, v)\|_{L^{p}\left(0, \tau ; \mathrm{D}\left(A_{D}^{1 / 4}\right)\right)} \leq C_{p}\|u\|_{L^{p}\left(0, \tau ; \mathrm{D}\left(A_{D}^{1 / 4}\right)\right)}\|v\|_{L^{\infty}\left(0, \tau ; \mathrm{D}\left(A_{D}^{1 / 4}\right)\right)} .
$$

If $v \in L^{\infty}\left(0, \tau ; V_{D}\right)$, the following improved estimate holds

$$
\|\phi(u, v)\|_{L^{p}\left(0, \tau ; \mathrm{D}\left(A_{D}^{\frac{1}{4}}\right)\right)} \leq K_{p} \tau^{\frac{1}{4}}\|u\|_{L^{p}\left(0, \tau ; \mathrm{D}\left(A_{D}^{1 / 4}\right)\right)}\|v\|_{L^{\infty}\left(0, \tau ; V_{D}\right)},
$$

where $K_{p}>0$ is a constant independent of $\tau$.

Proof. First, let $\mathcal{M}$ the maximal regularity operator on $H_{D}$ : for all $\varphi \in L^{p}\left(0, \tau ; H_{D}\right), \mathcal{M} \varphi$ is defined by

$$
\mathcal{M} \varphi(t):=\int_{0}^{t} A_{D} e^{-(t-s) A_{D}} \varphi(s) \mathrm{d} s, \quad t \in(0, \tau) .
$$

Since $H_{D}$ is a Hilbert space and $-A_{D}$ generates an analytic semigroup in $H_{D}$, the operator $\mathcal{M}$ is bounded on $L^{p}\left(0, \tau ; H_{D}\right)$ for all $p \in(1, \infty)$ and all $\tau>0$; see,e.g., [13]. Moreover, $\|\mathcal{M}\|_{\mathscr{L}\left(L^{p}\left(0, \tau ; H_{D}\right)\right)}$ is independent of $\tau$. Then

$$
A_{D}^{\frac{1}{4}} \phi(u, v)=\mathcal{M}\left(A_{D}^{-\frac{3}{4}} f\right)
$$


where $f$ is defined by (1.22). For $u \in L^{p}\left(0, \tau ; \mathrm{D}\left(A_{D}^{\frac{1}{4}}\right)\right.$ and $v \in L^{\infty}\left(0, \tau ; \mathrm{D}\left(A_{D}^{\frac{1}{4}}\right)\right.$, by Sobolev embeddings, $J u \otimes J v+J v \otimes J u \in L^{p}\left(0, \tau ; L^{3 / 2}\left(\Omega ; \mathbb{R}^{3}\right)\right)$, with the estimate

$$
\|J u \otimes J v+J v \otimes J u\|_{L^{p}\left(0, \tau ; L^{3 / 2}\left(\Omega ; \mathbb{R}^{3}\right)\right)} \leq C\|u\|_{L^{p}\left(0, \tau ; \mathrm{D}\left(A_{D}^{1 / 4}\right)\right)}\|v\|_{L^{\infty}\left(0, \tau ; \mathrm{D}\left(A_{D}^{1 / 4}\right)\right)},
$$

where the constant $C$ depends only on the constant of the embedding $\mathrm{D}\left(A_{D}^{\frac{1}{4}}\right) \hookrightarrow L^{3}\left(\Omega ; \mathbb{R}^{3}\right)$. This implies that $f \in L^{p}\left(0, \tau ; \mathbb{P}_{1}\left(W^{-1,3 / 2}\right)\right)$. Since $\mathrm{D}\left(A_{D}^{\frac{3}{4}}\right) \hookrightarrow W_{0}^{1,3}\left(\Omega ; \mathbb{R}^{3}\right)$ (see Proposition 1.6), the embedding $\mathbb{P}_{1}\left(W^{-1,3 / 2}\left(\Omega ; \mathbb{R}^{3}\right)\right) \hookrightarrow\left(\mathrm{D}\left(A_{D}^{\frac{3}{4}}\right)\right)^{\prime}$ holds and therefore $A_{D}^{-\frac{3}{4}} f \in$ $L^{p}\left(0, \tau ; H_{D}\right)$ with

$$
\left\|A_{D}^{-\frac{3}{4}} f\right\|_{L^{p}\left(0, \tau ; H_{D}\right)} \leq C\|u\|_{L^{p}\left(0, \tau ; \mathrm{D}\left(A_{D}^{1 / 4}\right)\right)}\|v\|_{L^{\infty}\left(0, \tau ; \mathrm{D}\left(A_{D}^{1 / 4}\right)\right)} .
$$

Using the $L^{p}$ maximal regularity result in $H_{D}$ gives (1.24).

To prove (1.25), let $u \in L^{p}\left(0, \tau ; \mathrm{D}\left(A_{D}^{\frac{1}{4}}\right)\right)$ and $v \in L^{\infty}\left(0, \tau ; V_{D}\right)$. Using the embeddings $\mathrm{D}\left(A_{D}^{\frac{1}{4}}\right) \hookrightarrow L^{3}\left(\Omega ; \mathbb{R}^{3}\right)$ and $V_{D} \hookrightarrow L^{6}\left(\Omega ; \mathbb{R}^{3}\right)$,

$$
\|J u \otimes J v+J v \otimes J u\|_{L^{p}\left(0, \tau ; L^{2}\left(\Omega, \mathbb{R}^{3}\right)\right)} \leq C\|u\|_{L^{p}\left(0, \tau ; \mathrm{D}\left(A_{D}^{1 / 4}\right)\right)}\|v\|_{L^{\infty}\left(0, \tau ; V_{D}\right)} .
$$

As before, this implies that $f \in L^{p}\left(0, \tau ; V_{D}^{\prime}\right)$ and therefore

$$
A_{D}^{\frac{1}{4}} \phi(u, v)(t)=\int_{0}^{t} A_{D}^{\frac{3}{4}} e^{(t-s) A_{D}}\left(A_{D}^{-\frac{1}{2}} f(s)\right) \mathrm{d} s, \quad t \in(0, \tau) .
$$

Using the analyticity of the semigroup $\left(e^{-t A_{D}}\right)_{t \geq 0}$ in $H_{D}$ and Young's inequality,

$$
\left\|A_{D}^{\frac{1}{4}} \phi(u, v)\right\|_{L^{p}\left(0, \tau ; H_{D}\right)} \leq C\left\|t \mapsto t^{-\frac{3}{4}}\right\|_{L^{1}(0, \tau)}\|u\|_{L^{p}\left(0, \tau ; \mathrm{D}\left(A_{D}^{1 / 4}\right)\right)}\|v\|_{L^{\infty}\left(0, \tau ; V_{D}\right)} .
$$

Proof of Theorem 1.11. The proof is inspired by the method described in [37] (see also [2, Section 8]). Let $p \in(1, \infty), \varepsilon>0$ to be chosen later and $w:=u-v \in \mathscr{C}_{b}\left(0, T ; \mathrm{D}\left(A_{D}^{\frac{1}{4}}\right)\right) \subset$ $L^{p}\left(0, T ; \mathrm{D}\left(A_{D}^{\frac{1}{4}}\right)\right): w$ satisfies

$$
\begin{aligned}
w & =\phi(u, w)+\phi(w, v)=\phi(w, u+v-2 \alpha)+2 \phi(w, \alpha) \\
& =\phi(w, u+v-2 \alpha)+2 \phi\left(w, \alpha-\alpha_{\varepsilon}\right)+2 \phi\left(w, \alpha_{\varepsilon}\right)
\end{aligned}
$$

where $\alpha_{\varepsilon}(t)=e^{-t A_{D}} u_{0, \varepsilon}$, with $u_{0, \varepsilon} \in V_{D}$ satisfying $\left\|u_{0, \varepsilon}-u_{0}\right\|_{\mathrm{D}\left(A_{D}^{1 / 4}\right)} \leq \varepsilon$. Using Lemma 1.12, $w$ is estimated in $L^{p}\left(0, \tau ; \mathrm{D}\left(A^{\frac{1}{4}}\right)\right)$ as follows

$$
\begin{aligned}
\|w\|_{L^{p}\left(0, \tau ; \mathrm{D}\left(A^{1 / 4}\right)\right)} & \leq\|w\|_{L^{p}\left(0, \tau ; \mathrm{D}\left(A^{1 / 4}\right)\right)}\left(C_{p}\left(\|u+v-2 \alpha\|_{L^{\infty}\left(0, \tau ; \mathrm{D}\left(A_{D}^{1 / 4}\right)\right)}+\varepsilon\right)+K_{p} \tau^{\frac{1}{4}}\left\|u_{0, \varepsilon}\right\|_{V_{D}}\right) \\
& \leq \kappa_{p}\left(\varepsilon+g_{\varepsilon}(\tau)\right)\|w\|_{L^{p}\left(0, \tau ; \mathrm{D}\left(A^{1 / 4}\right)\right)},
\end{aligned}
$$

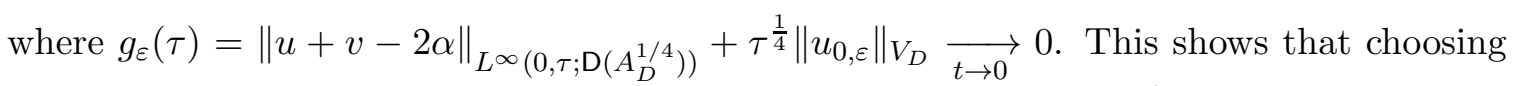
$\varepsilon>0$ small enough, there exists $\tau>0$ such that $\|w\|_{L^{p}\left(0, \tau ; \mathrm{D}\left(A^{1 / 4}\right)\right)} \leq \frac{1}{2}\|w\|_{L^{p}\left(0, \tau ; \mathrm{D}\left(A^{1 / 4}\right)\right)}$; in other terms, $w=0$ on $[0, \tau)$ (recall that $w$ is continuous on $[0, T)$ ). If $\tau=T$, then it was proved that $u=v$ on $[0, T)$. If $\tau<T$, by continuity, $w(\tau)=0$ also holds. The previous reasoning can be iterated on intervals of the form $[k \tau,(k+1) \tau)$ to prove ultimately that $w=0$ on $[0, T)$ (remark again that all constants $C_{p}, K_{p}, \kappa_{p}$ appearing in the estimates above are independent of $\tau$ ). 


\section{Neumann boundary conditions}

In this section, the system $\{(\mathrm{NS}),(\mathrm{Nbc})\}$ is studied. The results proved in [36] will be only surveyed, the method to prove existence of solutions being similar to what has been done in Section 1.

\subsection{The linear Neumann-Stokes operator}

Before defining the Neumann-Stokes operator, the following integration by parts formula will be useful.

Lemma 2.1. Let $\lambda \in \mathbb{R}, u, w: \Omega \rightarrow \mathbb{R}^{3}, \pi, \rho: \Omega \rightarrow \mathbb{R}$ sufficiently nice functions defined on the Lipschitz domain $\Omega \subset \mathbb{R}^{3}$. Let $L_{\lambda} u=\Delta u+\lambda \nabla(\operatorname{div} u)$ and define the conormal derivative

$$
\partial_{\nu}^{\lambda}(u, \pi)=\left(\lambda \nabla u+(\nabla u)^{\top}\right) \nu-\pi \nu \quad \text { on } \partial \Omega .
$$

Then the following integration by parts formula hold

$$
\begin{gathered}
\int_{\Omega}\left(L_{\lambda} u-\nabla \pi\right) \cdot w \mathrm{~d} x=-\int_{\Omega}\left[I_{\lambda}(\nabla u, \nabla w)-\pi \operatorname{div} w\right] \mathrm{d} x+\int_{\partial \Omega} \partial_{\nu}^{\lambda}(u, \pi) \cdot w \mathrm{~d} \sigma \\
=\int_{\Omega}\left(L_{\lambda} w-\nabla \rho\right) \cdot u \mathrm{~d} x+\int_{\Omega}[\pi \operatorname{div} w-\rho \operatorname{div} u] \mathrm{d} x \\
\quad+\int_{\partial \Omega}\left[\partial_{\nu}^{\lambda}(u, \pi) \cdot w-\partial_{\nu}^{\lambda}(w, \rho) \cdot u\right] \mathrm{d} \sigma,
\end{gathered}
$$

where

$$
I_{\lambda}(\xi, \zeta)=\sum_{i, j=1}^{3}\left(\xi_{i, j} \zeta_{i, j}+\lambda \xi_{i, j} \zeta_{j, i}\right), \quad \text { for } \xi=\left(\xi_{i, j}\right)_{1 \leq i, j \leq 3} \text { and } \zeta=\left(\zeta_{i, j}\right)_{1 \leq i, j \leq 3}
$$

Recall that $\nabla u=\left(\partial_{i} u_{j}\right)_{1 \leq i, j \leq 3}$.

The space $L^{2}\left(\Omega ; \mathbb{R}^{3}\right)$ admits the following Hodge decomposition, dual to the one shown in Section 1: $H_{N} \stackrel{\perp}{\oplus} G_{0}$, where $G_{0}:=\left\{\nabla \pi ; \pi \in H_{0}^{1}(\Omega ; \mathbb{R})\right\}$ and

$$
H_{N}:=\left\{u \in L^{2}\left(\Omega ; \mathbb{R}^{3}\right) ; \operatorname{div} u=0\right\} .
$$

Following the steps of the previous section, define $V_{N}=H^{1}\left(\Omega ; \mathbb{R}^{3}\right) \cap H_{N}$ and $J_{N}: H_{N} \hookrightarrow$ $L^{2}\left(\Omega ; \mathbb{R}^{3}\right)$ the canonical embedding, $\mathbb{P}_{N}=J_{N}^{\prime}: L^{2}\left(\Omega ; \mathbb{R}^{3}\right) \rightarrow H_{N}$ the orthogonal projection, $\tilde{J}_{N}: V_{N} \hookrightarrow H^{1}\left(\Omega ; \mathbb{R}^{3}\right)$ the restriction of $J_{N}$ on $V_{N}$ and $\tilde{J}_{N}^{\prime}=\tilde{\mathbb{P}}_{N}:\left(H^{1}\left(\Omega ; \mathbb{R}^{3}\right)\right)^{\prime} \rightarrow V_{N}^{\prime}$, extension of $\mathbb{P}_{N}$ to $\left(H^{1}\left(\Omega ; \mathbb{R}^{3}\right)\right)^{\prime}$. The Neumann-Stokes operator is defined as follows.

Definition 2.2. Let $\lambda \in \mathbb{R}$. The Neumann-Stokes operator $A_{\lambda}$ is defined as being the associated operator of the bilinear form

$$
a_{\lambda}: V_{N} \times V_{N} \rightarrow \mathbb{R}, \quad a_{\lambda}(u, v)=\int_{\Omega} I_{\lambda}\left(\nabla \tilde{J}_{N} u, \nabla \tilde{J}_{N} v\right) d x
$$

In the case where $\lambda \in(-1,1]$, the bilinear form $a_{\lambda}$ is continuous, symmetric, coercive and sectorial. So its associated operator is self-adjoint, invertible and the negative generator of an analytic semigroup of contractions on $H_{N}$.

The following proposition is a consequence of the integration by parts formula (2.2), [36, Theorem 6.8] and [28, Théorème 5.3]. 
Proposition 2.3. Let $\lambda \in(-1,1]$. The Neumann-Stokes operator $A_{\lambda}$ is the part in $H_{N}$ of the bounded operator $A_{0, \lambda}: V_{N} \rightarrow V_{N}^{\prime}$ defined by $\left(A_{0, \lambda} u\right)(v)=a_{\lambda}(u, v)$. The operator $A_{\lambda}$ is self-adjoint, invertible, $-A_{\lambda}$ generates an analytic semigroup of contractions on $H_{N}$, $\mathrm{D}\left(A_{\lambda}^{\frac{1}{2}}\right)=V_{N}$ and for all $u \in \mathrm{D}\left(A_{\lambda}\right)$, there exists $\pi \in L^{2}(\Omega ; \mathbb{R})$ such that

$$
J_{N} A_{\lambda} u=-\Delta \tilde{J}_{N} u+\nabla \pi
$$

and $\mathrm{D}\left(A_{\lambda}\right)$ admits the following description

$$
\mathrm{D}\left(A_{\lambda}\right)=\left\{u \in V_{N} ; \exists \pi \in L^{2}(\Omega ; \mathbb{R}): f=-\Delta \tilde{J}_{N} u+\nabla \pi \in H_{N} \text { and } \partial_{\nu}^{\lambda}(u, \pi)_{f}=0\right\},
$$

where $\partial_{\nu}^{\lambda}(u, \pi)_{f}$ is defined in a weak sense for all $f \in\left(H^{1}\left(\Omega ; \mathbb{R}^{3}\right)\right)^{\prime}$ by

$$
\left\langle\partial_{\nu}^{\lambda}(u, \pi)_{f}, \psi\right\rangle_{\partial \Omega}={ }_{\left(H^{1}\right)^{\prime}}\langle f, \Psi\rangle_{H^{1}}+\int_{\Omega} I_{\lambda}\left(\nabla \tilde{J}_{n} u, \nabla \Psi\right) \mathrm{d} x-{ }_{L^{2}}\langle\pi, \operatorname{div} \Psi\rangle_{L^{2}}
$$

for $\Psi \in H^{1}(\Omega)$ and $\psi=\operatorname{Tr}_{\partial \Omega} \Psi$.

Remark 2.4. If $f \in\left(H^{1}\left(\Omega ; \mathbb{R}^{3}\right)\right)^{\prime}$, the quantity $\partial_{\nu}^{\lambda}(u, \pi)_{f}$ exists on $\partial \Omega$ in the Besov space $B_{-\frac{1}{2}}^{2,2}\left(\partial \Omega ; \mathbb{R}^{3}\right)=H^{-\frac{1}{2}}\left(\partial \Omega, \mathbb{R}^{3}\right)$ according to [36, Proposition 3.6].

Thanks to [36, Sections $9 \& 10]$, a good description of the domain of fractional powers of the Neumann-Stokes operator $A_{\lambda}$ can be given. In particular, in [36, Corollary 10.6] it was established that

$$
\mathrm{D}\left(A_{\lambda}^{\frac{3}{4}}\right) \text { is continuously embedded into } W^{1,3}\left(\Omega ; \mathbb{R}^{3}\right) .
$$

\subsection{The nonlinear Neumann-Navier-Stokes equations}

The results in 2.1 allow to prove a result similar to Theorem 1.10 for the system $\{(\mathrm{NS}),(\mathrm{Nbc})\}$. As in the previous section, it is not difficult to see that $\mathrm{D}\left(A_{\lambda}^{\frac{1}{4}}\right) \hookrightarrow L^{3}\left(\Omega ; \mathbb{R}^{3}\right)$ is a critical space for the system. For $T \in(0, \infty]$, following the definition of $\mathscr{E}_{T}$ in Section 1, define

$$
\begin{aligned}
\mathscr{F}_{T}= & \left\{u \in \mathscr{C}_{b}\left([0, T] ; \mathrm{D}\left(A_{\lambda}^{\frac{1}{4}}\right)\right) ; u(t) \in \mathrm{D}\left(A_{\lambda}^{\frac{3}{4}}\right), u^{\prime}(t) \in \mathrm{D}\left(A_{\lambda}^{\frac{1}{4}}\right) \text { for all } t \in(0, T]\right. \\
& \text { and } \left.\sup _{t \in(0, T)}\left\|t^{\frac{1}{2}} A_{\lambda}^{\frac{3}{4}} u(t)\right\|_{2}+\sup _{t \in(0, T)}\left\|t A_{\lambda}^{\frac{1}{4}} u^{\prime}(t)\right\|_{2}<\infty\right\}
\end{aligned}
$$

endowed with the norm

$$
\|u\|_{\mathscr{F}_{T}}=\sup _{t \in(0, T)}\left\|A_{\lambda}^{\frac{1}{4}} u(t)\right\|_{2}+\sup _{t \in(0, T)}\left\|t^{\frac{1}{2}} A_{\lambda}^{\frac{3}{4}} u(t)\right\|_{2}+\sup _{t \in(0, T)}\left\|t A_{\lambda}^{\frac{1}{4}} u^{\prime}(t)\right\|_{2} .
$$

The same tools as in 1.2 apply, so the following result can be proved (see [36, Theorem 11.3]).

Theorem 2.5. Let $\Omega \subset \mathbb{R}^{3}$ be a bounded Lipschitz domain and let $u_{0} \in \mathrm{D}\left(A_{\lambda}^{\frac{1}{4}}\right)$. Let $\beta$ and $\psi$ be defined by

$$
\beta(t)=e^{-t A_{\lambda}} u_{0}, \quad t \geq 0,
$$

and for $u, v \in \mathscr{F}_{T}$ and $t \in(0, T)$,

$$
\left.\psi(u, v)(t)=\int_{0}^{t} e^{-(t-s) A_{\lambda}}\left(-\frac{1}{2} \mathbb{P}_{N}\right)\left(\left(J_{N} u(s) \cdot \nabla\right) \tilde{J}_{N} v(s)+J_{N} v(s) \cdot \nabla\right) \tilde{J}_{M} u(s)\right) \mathrm{d} s .
$$


(i) If $\left\|A_{\lambda}^{\frac{1}{4}} u_{0}\right\|_{2}$ is small enough, then there exists a unique $u \in \mathscr{F}_{\infty}$ solution of $u=$ $\beta+\psi(u, u)$.

(ii) For all $u_{0} \in \mathrm{D}\left(A_{\lambda}^{\frac{1}{4}}\right)$, there exists $T>0$ and a unique $u \in \mathscr{F}_{T}$ solution of $u=$ $\beta+\psi(u, u)$.

A comment here may be necessary to link the solution $u$ obtained in Theorem 2.5 and a solution of the system $\{(\mathrm{NS}),(\mathrm{Nbc})\}$. If $u \in \mathcal{F}_{T}$, then $u^{\prime} \in H_{N}$ and $\left(J_{N} u \cdot \nabla\right) \tilde{J}_{N} u \in$ $L^{2}\left(\Omega ; \mathbb{R}^{n}\right)$. Moreover, if $u$ satisfies the equation $u=\beta+\psi(u, u)$, then $u$ is a mild solution of

$$
A_{\lambda} u=-u^{\prime}-\mathbb{P}_{N}\left(\left(J_{N} u \cdot \nabla\right) \tilde{J}_{N} u\right) \in H_{N} .
$$

Going further,

$$
J_{N} \mathbb{P}_{N}\left(\left(J_{N} u \cdot \nabla\right) \tilde{J}_{N} u\right)=\left(J_{N} u \cdot \nabla\right) \tilde{J}_{N} u-\nabla q
$$

where $q \in H_{0}^{1}(\Omega ; \mathbb{R})$ satisfies

$$
\left.\Delta q=\operatorname{div}\left(J_{N} u \cdot \nabla\right) \tilde{J}_{N} u\right) \in H^{-1}\left(\Omega ; \mathbb{R}^{n}\right) .
$$

Therefore, by definition of $A_{\lambda}$, there exists $\pi \in L^{2}(\Omega, \mathbb{R})$ such that

$$
-\Delta \tilde{J}_{n} u+\nabla \pi=J_{N}\left(A_{\lambda} u\right)=-J_{N} u^{\prime}-\left(J_{N} u \cdot \nabla\right) \tilde{J}_{N} u+\nabla q
$$

and at the boundary, $(u, \pi)$ satisfies $(\mathrm{Nbc})$ in the weak sense as in Proposition 2.3. Since $q \in H_{0}^{1}(\Omega ; \mathbb{R}),(u, \pi-q)$ satisfies also $(\mathrm{Nbc})$. This proves that $(u, \pi-q)$ is a solution of the system $\{(\mathrm{NS}),(\mathrm{Nbc})\}$.

The uniqueness is true in a larger space than $\mathscr{F}_{T}$ : for each $u_{0} \in \mathrm{D}\left(A^{\frac{1}{4}}\right)$, there is at most one $u \in \mathscr{C}_{b}\left([0, T) ; \mathrm{D}\left(A^{\frac{1}{4}}\right)\right)$, mild solution of the system $\{(\mathrm{NS}),(\mathrm{Nbc})\}$. For a more precise statement, see [36, Theorem 11.8].

\section{Hodge boundary conditions}

Most of the results presented here are proved thoroughly in [35] for the linear theory and [34] for the nonlinear system. The linear Hodge-Laplacian on $L^{p}$-spaces is first studied and then the Hodge-Stokes operator before applying the properties of this operator to prove the existence of mild solutions of the Hodge-Navier-Stokes system in $L^{3}$. Some recent developments/improvements can be found in [29].

\subsection{The Hodge-Laplacian and the Hodge-Stokes operators}

We denote by $H$ the space $L^{2}\left(\Omega ; \mathbb{R}^{3}\right)$. Let

$$
\begin{aligned}
& W_{T}:=\left\{u \in H ; \operatorname{curl} u \in H, \operatorname{div} u \in L^{2}(\Omega ; \mathbb{R}) \text { and } \nu \cdot u=0 \text { on } \partial \Omega\right\}, \\
& \text { and } \quad W_{N}:=\left\{u \in H ; \operatorname{curl} u \in H, \operatorname{div} u \in L^{2}(\Omega ; \mathbb{R}) \text { and } \nu \times u=0 \text { on } \partial \Omega\right\},
\end{aligned}
$$

(subscript $T$ is for "tangential" and $N$ for "normal") both endowed with the scalar product

$$
\left\langle\langle u, v\rangle_{W}:=\langle\operatorname{curl} u, \operatorname{curl} v\rangle_{\Omega}+\langle\operatorname{div} u, \operatorname{div} v\rangle_{\Omega}+\langle u, v\rangle_{\Omega},\right.
$$

where $\langle\cdot, \cdot\rangle_{E}$ denotes the $L^{2}(E)$-pairing. 
Remark 3.1. As in Remark 1.1 for a bounded Lipschitz domain $\Omega$ and a vector field $w \in H$ satisfying curl $w \in H$, define $\nu \times w$ on $\partial \Omega$ in the following weak sense in $H^{-\frac{1}{2}}\left(\partial \Omega ; \mathbb{R}^{3}\right)$ : for $\phi \in H^{1}\left(\Omega ; \mathbb{R}^{3}\right)$,

$$
\langle\operatorname{curl} w, \phi\rangle_{\Omega}-\langle w, \operatorname{curl} \phi\rangle_{\Omega}=\langle\nu \times w, \phi\rangle_{\partial \Omega}
$$

where $\varphi=\operatorname{Tr}_{\left.\right|_{\partial} \Omega} \phi$, the right hand-side of (3.1) depends only on $\varphi$ on $\partial \Omega$ and not on the choice of $\phi$, its extension to $\Omega$.

Remark 3.2. In the case of smooth bounded domains, i.e., with a $\mathscr{C}^{1,1}$ boundary or convex, the spaces $W_{T}$ and $W_{N}$ are contained in $H^{1}\left(\Omega ; \mathbb{R}^{3}\right)$ (see, e.g., [3, Theorems 2.9, 2.12 and 2.17]).

This is not the case if $\Omega$ is only Lipschitz. The Sobolev embedding associated to the spaces $W_{T, N}$ is as follows: $W_{T, N} \hookrightarrow H^{\frac{1}{2}}\left(\Omega ; \mathbb{R}^{3}\right)$ with the estimate

$$
\|u\|_{H^{1 / 2}} \leq C\left[\|u\|_{2}+\|\operatorname{curl} u\|_{2}+\|\operatorname{div} u\|_{2}\right], \quad u \in W_{T, N} ;
$$

see for instance [9] or [31, Theorem 11.2] where it was proved moreover that

$$
\begin{aligned}
& \text { if } u \in W_{T, N} \text {, then } u \text { has an } L^{2} \text { trace at the boundary } \partial \Omega \text { : } \\
& u_{\mid \partial \Omega}=(\nu \cdot u) \nu+(\nu \times u) \times \nu \in L^{2}\left(\partial \Omega ; \mathbb{R}^{3}\right), \\
& \text { and }\left\|u_{\mid \partial \Omega}\right\|_{L^{2}\left(\partial \Omega ; \mathbb{R}^{3}\right)} \leq C\left[\|u\|_{2}+\|\operatorname{curl} u\|_{2}+\|\operatorname{div} u\|_{2}\right] .
\end{aligned}
$$

Remark 3.3. If $\Omega$ is of class $\mathscr{C}^{1}$, the previous result applies also if $u \in L^{p}\left(\Omega ; \mathbb{R}^{3}\right)$ with $\operatorname{curl} u \in L^{p}\left(\Omega ; \mathbb{R}^{3}\right), \operatorname{div} u \in L^{p}(\Omega ; \mathbb{R})$, and $\nu \cdot u=0$ on $\partial \Omega($ or $\nu \times u=0$ on $\partial \Omega$ ) if $p \in(1, \infty)$ (see [31, Theorem 11.2], where it was proved that if $\Omega$ is only Lipschitz, it is also true for $p$ in a range around 2).

Remark 3.4. The Helmholtz projection $\mathbb{P}: L^{2}\left(\Omega ; \mathbb{R}^{3}\right) \rightarrow H_{D}$ defined in Section 1 (after Remark 1.3) maps also $W_{T}$ to the space $\left\{u \in W_{T} ; \operatorname{div} u=0\right\}=: \mathcal{V}_{T}$.

The projection $\mathbb{P}_{N}: L^{2}\left(\Omega ; \mathbb{R}^{3}\right) \rightarrow H_{N}$ defined in Section 2 (before Definition 2.2) maps also $W_{N}$ to the space $\left\{u \in W_{N} ; \operatorname{div} u=0\right\}=: \mathcal{V}_{N}$.

On $W_{T} \times W_{T}$, we define the following form

$$
b_{T}: W_{T} \times W_{T} \rightarrow \mathbb{R}, \quad b_{T}(u, v)=\langle\operatorname{curl} u, \operatorname{curl} v\rangle+\langle\operatorname{div} u, \operatorname{div} v\rangle,
$$

where $\langle\cdot, \cdot\rangle$ denotes either the scalar or the vector-valued $L^{2}$-pairing. Similarly, we define

$$
b_{N}: W_{N} \times W_{N} \rightarrow \mathbb{R}, \quad b_{N}(u, v)=\langle\operatorname{curl} u, \operatorname{curl} v\rangle+\langle\operatorname{div} u, \operatorname{div} v\rangle .
$$

Proposition 3.5. The Hodge-Laplacian operators $B_{T}$ and $B_{N}$, defined as the associated operators in $H$ of the forms $b_{T}$ and $b_{N}$, satisfy

$$
\begin{aligned}
\mathrm{D}\left(B_{T, N}\right) & =\left\{u \in W_{T, N} ; \nabla \operatorname{div} u \in H, \operatorname{curl} \operatorname{curl} u \in H \text { and } \mid \begin{array}{l}
\nu \times \operatorname{curl} u \\
(\operatorname{div} u) \nu
\end{array}=0 \text { on } \partial \Omega\right\} \\
B_{T, N} u & =-\Delta u, \quad u \in \mathrm{D}\left(B_{T, N}\right) .
\end{aligned}
$$

Proof. Let $u \in W_{T, N}$ and $v \in H_{0}^{1}\left(\Omega ; \mathbb{R}^{3}\right) \subset W_{T, N}$. Then

$$
b_{T, N}(u, v)={ }_{H^{-1}}\langle-\nabla \operatorname{div} u+\operatorname{curl} \operatorname{curl} u, v\rangle_{H_{0}^{1}}={ }_{H^{-1}}\langle-\Delta u, v\rangle_{H_{0}^{1}}
$$


so that $B_{T, N} u=-\Delta u$ in $H^{-1}\left(\Omega ; \mathbb{R}^{3}\right)$.

The proof of Proposition 3.5 is described now in the case of $b_{T}$ defined on $W_{T} \times W_{T}$. The case of $b_{N}$ defined on $W_{N} \times W_{N}$ can be proved with the same arguments (using $\mathbb{P}_{N}$ instead of $\mathbb{P}$ in what follows). Let $D$ be the space

$$
D:=\left\{u \in W_{T} ; \nabla \operatorname{div} u \in H, \operatorname{curl} \operatorname{curl} u \in H \text { and } \nu \times \operatorname{curl} u=0 \text { on } \partial \Omega\right\} .
$$

If $u \in D$, then $B_{T} u=-\Delta u \in H$ and therefore $u \in \mathrm{D}\left(B_{T}\right)$.

Conversely, assume that $u \in \mathrm{D}\left(B_{T}\right)$. Then $(\mathrm{Id}-\mathbb{P}) B_{T} u \in H$ satisfies for all $v \in W_{T}$

$$
\begin{aligned}
\left\langle(\operatorname{Id}-\mathbb{P}) B_{T} u, v\right\rangle & =\left\langle B_{T} u,(\operatorname{Id}-\mathbb{P}) v\right\rangle=b_{T}(u, v)-b_{T}(u, \mathbb{P} v) \\
& =\langle\operatorname{div} u, \operatorname{div} v\rangle={ }_{W_{T}^{\prime}}\langle-\nabla \operatorname{div} u, v\rangle_{W_{T}},
\end{aligned}
$$

so that $-\nabla \operatorname{div} u=(\operatorname{Id}-\mathbb{P}) B_{T} u \in H$. Then $\operatorname{curl} \operatorname{curl} u=B_{T} u+\nabla \operatorname{div} u \in H$. It remains to prove that $\nu \times \operatorname{curl} u=0$ on $\partial \Omega$. Remark that it makes sense to consider the tangential part of $w:=\operatorname{curl} u$ on the boundary $\partial \Omega$ since it was just proved that curl $w \in H$ and therefore, thanks to (3.1), $\nu \times w \in H^{-\frac{1}{2}}\left(\partial \Omega ; \mathbb{R}^{3}\right)$. For all $\varphi \in H^{\frac{1}{2}}\left(\partial \Omega ; \mathbb{R}^{3}\right) \cap L_{\text {tan }}^{2}\left(\partial \Omega ; \mathbb{R}^{3}\right)$, there exists $\phi \in H^{1}\left(\Omega ; \mathbb{R}^{3}\right)$ such that $\phi_{l_{\partial \Omega}}=\varphi$. In that case, $\phi \in W_{T}$ and therefore

$$
\begin{aligned}
\langle-\nabla \operatorname{div} u+\operatorname{curl} \operatorname{curl} u, \phi\rangle & =\left\langle B_{T} u, \phi\right\rangle=b_{T}(u, \phi) \\
& =\langle\operatorname{div} u, \operatorname{div} \phi\rangle+\langle\operatorname{curl} u, \operatorname{curl} \phi\rangle \\
& =\langle-\nabla \operatorname{div} u+\operatorname{curl} \operatorname{curl} u, \phi\rangle-{ }_{H^{-1 / 2}(\partial \Omega)}\langle\nu \times \operatorname{curl} u, \varphi\rangle_{H^{1 / 2}(\partial \Omega)} .
\end{aligned}
$$

It proves that $H^{-1 / 2}(\partial \Omega)\langle\nu \times \operatorname{curl} u, \varphi\rangle_{H^{1 / 2}(\partial \Omega)}=0$ for all $\varphi \in H^{\frac{1}{2}}\left(\partial \Omega ; \mathbb{R}^{3}\right) \cap L_{\text {tan }}^{2}\left(\partial \Omega ; \mathbb{R}^{3}\right)$, and then $\nu \times \operatorname{curl} u=0$ on $\partial \Omega$.

Since the forms $b_{T, N}$ are continuous, bilinear, symmetric, coercive and sectorial, the operators $-B_{T, N}$ generate analytic semigroups of contractions on $H, B_{T, N}$ is self-adjoint and $\mathrm{D}\left(B_{T, N}^{1 / 2}\right)=W_{T, N}$. The following property will be useful in next Section; it links $B_{T}$ and $B_{N}$, as shown in [41, Proposition 2.2].

Lemma 3.6. For $u \in H$ such that $\operatorname{curl} u \in H$, the following commutator property occurs for all $\varepsilon>0$

$$
\operatorname{curl}\left(1+\varepsilon B_{T}\right)^{-1} u=\left(1+\varepsilon B_{N}\right)^{-1} \operatorname{curl} u .
$$

Proof. Let $u \in H$ such that $\operatorname{curl} u \in H$. Let $u_{\varepsilon}=\left(1+\varepsilon B_{T}\right)^{-1} u$ and $w_{\varepsilon}=\left(1+\varepsilon B_{N}\right)^{-1} \operatorname{curl} u$. Step 1: $\operatorname{curl} u_{\varepsilon} \in D\left(B_{N}\right)$.

By (3.5), it holds curl $u_{\varepsilon} \in H, \operatorname{curl} \operatorname{curl} u_{\varepsilon} \in H, \operatorname{div}\left(\operatorname{curl} u_{\varepsilon}\right)=0 \in H^{1}(\Omega), \nu \times \operatorname{curl} u_{\varepsilon}=0$ on $\partial \Omega$ and $\operatorname{div}\left(\operatorname{curl} u_{\varepsilon}\right)=0$ on $\partial \Omega$. To prove that $\operatorname{curl} u_{\varepsilon} \in D\left(B_{T}\right)$, it remains to show, thanks to (3.5), that $\operatorname{curl} \operatorname{curl}\left(\operatorname{curl} u_{\varepsilon}\right) \in H$. This is due to the fact that

$$
\operatorname{curl} \operatorname{curl}\left(\operatorname{curl} u_{\varepsilon}\right)=\operatorname{curl}\left(-\Delta u_{\varepsilon}\right) \quad \text { in } H^{-1}\left(\Omega, \mathbb{R}^{3}\right) .
$$

Since

$$
-\Delta u_{\varepsilon}=B_{T}\left(1+\varepsilon B_{T}\right)^{-1} u=\frac{1}{\varepsilon}\left(u-u_{\varepsilon}\right)
$$

and $\operatorname{curl} u_{\varepsilon}, \operatorname{curl} u \in H$, the claim follows.

Step 2: $\operatorname{curl} u_{\varepsilon}=w_{\varepsilon}$. 
By Step $1, \operatorname{curl} u_{\varepsilon} \in D\left(B_{N}\right)$. Moreover, in the sense of distributions

$$
\left(1+\varepsilon B_{N}\right)\left(\operatorname{curl} u_{\varepsilon}\right)=\operatorname{curl} u_{\varepsilon}-\varepsilon \Delta \operatorname{curl} u_{\varepsilon}=\operatorname{curl}\left(u_{\varepsilon}-\varepsilon \Delta u_{\varepsilon}\right)=\operatorname{curl} u
$$

since $u_{\varepsilon}-\varepsilon \Delta u_{\varepsilon}=\left(1+\varepsilon B_{T}\right)\left(1+\varepsilon B_{T}\right)^{-1} u=u$. Therefore,

$$
\operatorname{curl} u_{\varepsilon}=\left(1+\varepsilon B_{N}\right)^{-1} \operatorname{curl} u=w_{\varepsilon}
$$

which proves the claim.

To prove that the operators $B_{T, N}$ extend to $L^{p}$-spaces, it suffices to prove that their resolvents admit $L^{2}-L^{2}$ off-diagonal estimates. This was proved in, e.g., [35, Section 6] (see also [29]).

Proposition 3.7. There exist two constants $C, c>0$ such that for any open sets $E, F \subset \mathbb{R}^{3}$ such that $\operatorname{dist}(E, F)>0$ and for all $t>0, f \in H$ and

$$
u=\left(\operatorname{Id}+t^{2} B_{T, N}\right)^{-1}\left(1_{F} f\right),
$$

it holds

$$
\left\|1_{E} u\right\|_{2}+t\left\|1_{E} \operatorname{div} u\right\|_{2}+t\left\|11_{E} \operatorname{curl} u\right\|_{2} \leq C e^{-c \frac{\operatorname{dist}(E, F)}{t}}\left\|1_{F} f\right\|_{2} .
$$

Proof. Start by choosing a smooth cut-off function $\xi: \mathbb{R}^{3} \rightarrow \mathbb{R}$ satisfying $\xi=1$ on $E, \xi=0$ on $F$ and $\|\nabla \xi\|_{\infty} \leq \frac{k}{\operatorname{dist}(E, F)}$. Then define $\eta=e^{\delta \xi}$ where $\delta>0$ is to be chosen later. Next, take the scalar product of the equation

$$
u-t^{2} \Delta u=1_{F} f, \quad u \in \mathrm{D}\left(B_{T, N}\right)
$$

with the function $v=\eta^{2} u$. Since $\eta=1$ on $F$ and $\|u\|_{2} \leq\left\|1_{F} f\right\|_{2}$, it is easy to check then that

$$
\begin{aligned}
&\|\eta u\|_{2}^{2}+t^{2}\|\eta \operatorname{div} u\|_{2}^{2}+t^{2}\|\eta \operatorname{curl} u\|_{2}^{2} \\
& \leq\left\|1_{F} f\right\|_{2}^{2}+2 \alpha\|\nabla \xi\|_{\infty} t^{2}\|\eta u\|_{2}\left(\|\eta \operatorname{div} u\|_{2}+\|\eta \operatorname{curl} u\|_{2}\right)
\end{aligned}
$$

and therefore, using the estimate on $\|\nabla \xi\|_{\infty}$ and choosing $\delta=\frac{\operatorname{dist}(E, F)}{4 k t}$,

$$
\|\eta u\|_{2}^{2}+t^{2}\|\eta \operatorname{div} u\|_{2}^{2}+t^{2}\|\eta \operatorname{curl} u\|_{2}^{2} \leq 2\left\|1_{F} f\right\|_{2}^{2} .
$$

Using now the fact that $\eta=e^{\delta}$ on $E$,

$$
\left\|1_{E} u\right\|_{2}+t\left\|1_{E} \operatorname{div} u\right\|_{2}+t\left\|1_{E} \operatorname{curl} u\right\|_{2} \leq \sqrt{2} e^{-\frac{\operatorname{dist}(E, F)}{4 k t}}\left\|1_{F} f\right\|_{2},
$$

which gives (3.7) with $C=\sqrt{2}$ and $c=\frac{1}{4 k}$.

With a slight modification of the proof, it can be shown that for all $\theta \in(0, \pi)$ there exist two constants $C, c>0$ such that for any open sets $E, F \subset \mathbb{R}^{3}$ such that dist $(E, F)>0$ and for all $z \in \Sigma_{\pi-\theta}=\{\omega \in \mathbb{C} \backslash\{0\} ;|\arg z|<\pi-\theta\}, f \in H$ and

$$
u=\left(z \operatorname{Id}+B_{T, N}\right)^{-1}\left(1_{F} f\right),
$$

it holds

$$
|z|\left\|11_{E} u\right\|_{2}+|z|^{\frac{1}{2}}\left\|1_{E} \operatorname{div} u\right\|_{2}+|z|^{\frac{1}{2}}\left\|1_{E} \operatorname{curl} u\right\|_{2} \leq C e^{-c \operatorname{dist}(E, F)|z|^{\frac{1}{2}}}\left\|1_{F} f\right\|_{2} .
$$


Following [30] and [10] (see also [29]), there exist Bogovskiu type operators $R_{i}, T_{i}, i=1,2,3$, and $K_{1,2}, L_{1,2}$ such that for all $p \in(1, \infty)$,

$$
\begin{aligned}
& R_{1}: L^{p}\left(\Omega ; \mathbb{R}^{3}\right) \rightarrow W^{1, p}(\Omega ; \mathbb{R}), \quad T_{1}: L^{p}\left(\Omega ; \mathbb{R}^{3}\right) \rightarrow W_{0}^{1, p}(\Omega ; \mathbb{R}), \\
& R_{2}: L^{p}\left(\Omega ; \mathbb{R}^{3}\right) \rightarrow W^{1, p}\left(\Omega ; \mathbb{R}^{3}\right), \quad \\
& T_{2}: L^{p}\left(\Omega ; \mathbb{R}^{3}\right) \rightarrow W_{0}^{1, p}\left(\Omega ; \mathbb{R}^{3}\right), \\
& R_{3}: L^{p}(\Omega ; \mathbb{R}) \rightarrow W^{1, p}\left(\Omega ; \mathbb{R}^{3}\right), \quad T_{3}: L^{p}(\Omega ; \mathbb{R}) \rightarrow W_{0}^{1, p}\left(\Omega ; \mathbb{R}^{3}\right), \\
& K_{1,2}: L^{p}\left(\Omega ; \mathbb{R}^{3}\right) \rightarrow W^{1, p}\left(\Omega ; \mathbb{R}^{3}\right), \quad \text { and } L_{1,2}: L^{p}\left(\Omega ; \mathbb{R}^{3}\right) \rightarrow W_{0}^{1, p}\left(\Omega ; \mathbb{R}^{3}\right)
\end{aligned}
$$

satisfying

$$
\begin{aligned}
& R_{2} \operatorname{curl} u+\nabla R_{1} u=u-K_{1} u \quad \forall u \in L^{p}\left(\Omega ; \mathbb{R}^{3}\right) \text { with } \operatorname{curl} u \in L^{p}(\Omega ; \mathbb{R}) \\
& \text { and } \operatorname{curl} K_{1} u=0 \text { if } \operatorname{curl} u=0, \\
& R_{3} \operatorname{div} u+\operatorname{curl} R_{2} u=u-K_{2} u, \quad \forall u \in L^{p}\left(\Omega ; \mathbb{R}^{3}\right) \text { with } \operatorname{div} u \in L^{p}(\Omega ; \mathbb{R}) \\
& \text { and } \operatorname{div} K_{2} u=0 \text { if } \operatorname{div} u=0, \\
& T_{2} \operatorname{curl} u+\nabla T_{1} u=u-L_{1} u, \quad \forall u \in L^{p}\left(\Omega ; \mathbb{R}^{3}\right) \text { with } \operatorname{curl} u \in L^{p}(\Omega ; \mathbb{R}), \\
& \nu \times u=0 \text { on } \partial \Omega \text { and } \operatorname{curl} L_{1} u=0 \text { if } \operatorname{curl} u=0, \\
& T_{3} \operatorname{div} u+\operatorname{curl} T_{2} u=u-L_{2} u, \quad \forall u \in L^{p}\left(\Omega ; \mathbb{R}^{3}\right) \text { with } \operatorname{div} u \in L^{p}(\Omega ; \mathbb{R}), \\
& \nu \cdot u=0 \text { on } \partial \Omega \text { and } \operatorname{div} L_{2} u=0 \text { if } \operatorname{div} u=0 .
\end{aligned}
$$

With these potential operators (at this point, only the relations (3.10) and (3.12) are needed) and (3.8), it is easy to prove that (see, e.g., [29])

$$
z\left(z \operatorname{Id}+B_{T}\right)^{-1} \text { is bounded in } H_{D}^{p} \text { and in } G_{p} \text { for } p \in\left[\frac{6}{5}, 2\right] \text { uniformly in } z \in \Sigma_{\pi-\theta}
$$

where $H_{D}^{p}:=\left\{u \in L^{p}\left(\Omega ; \mathbb{R}^{3}\right)\right.$ s.t. $\operatorname{div} u=0$ and $\nu \cdot u=0$ on $\left.\partial \Omega\right\}$ and $G_{p}:=\nabla W^{1, p}(\Omega ; \mathbb{R})$ are defined for $p \in(1, \infty)$; if $p=2$, then $H_{D}^{2}=H_{D}$ and $G_{2}=G$ defined in Section 1. With the same reasoning, one can prove that

$$
z\left(z \operatorname{Id}+B_{N}\right)^{-1} \text { is bounded in } H_{N}^{p} \text { and in } G_{p, 0} \text { for } p \in\left[\frac{6}{5}, 2\right] \text { uniformly in } z \in \Sigma_{\pi-\theta}
$$

where $H_{N}^{p}:=\left\{u \in L^{p}\left(\Omega ; \mathbb{R}^{3}\right)\right.$ s.t. $\left.\operatorname{div} u=0\right\}$ and $G_{p, 0}:=\nabla W_{0}^{1, p}(\Omega ; \mathbb{R})$ are defined for $p \in(1, \infty)$; if $p=2$, then $H_{N}^{2}=H_{N}$ and $G_{2,0}=G_{0}$ defined in Section 2.

Proposition 3.8. The resolvents $\left\{z\left(z \mathrm{Id}+B_{T, N}\right)^{-1}, z \in \Sigma_{\pi-\theta}\right\}$ are uniformly bounded in $L^{p}\left(\Omega ; \mathbb{R}^{3}\right)$ for all $p \in\left(q_{0}^{\prime}, q_{0}\right)$, where $q_{0}:=\min \{6,3+\varepsilon\} \quad(\varepsilon>0$ depends on $\partial \Omega)$.

Proof. By [19, Theorems 11.1 and 11.2], the projections defined in Section 1 and Section 2

$$
\mathbb{P} \text { and } \mathbb{P}_{N} \text { extend to bounded projections on } L^{p}\left(\Omega ; \mathbb{R}^{3}\right) \text { for } p \in\left((3+\varepsilon)^{\prime}, 3+\varepsilon\right),
$$

where $\varepsilon>0$ depends on $\partial \Omega$ (and $\left.(3+\varepsilon)^{\prime}=\frac{3+\varepsilon}{2+\varepsilon}<\frac{3}{2}\right)$; if $\Omega$ is of class $\mathscr{C}^{1}$, then $\varepsilon=\infty$. This means in particular that $H_{D}^{p}$ coincides with the space $L_{\sigma}^{p}(\Omega)$ defined in (1.4) for all $p \in\left((3+\varepsilon)^{\prime}, 3+\varepsilon\right)$. Therefore for all $p \in\left(q_{0}^{\prime}, 2\right]$, the resolvents $\left\{z\left(z \operatorname{Id}+B_{T, N}\right)^{-1}, z \in \Sigma_{\pi-\theta}\right\}$ are uniformly bounded in $L^{p}\left(\Omega ; \mathbb{R}^{3}\right)$. The same result for all $p \in\left[2, q_{0}\right)$ is obtained by duality. 
Corollary 3.9. The semigroups $\left(e^{-t B_{T, N}}\right)_{t \geq 0}$ extend to bounded analytic semigroups on $L^{p}\left(\Omega ; \mathbb{R}^{3}\right)$ for $p \in\left(q_{0}^{\prime}, q_{0}\right)$ and satisfy

$$
\begin{array}{ll}
\left\|\sqrt{t} \operatorname{div}\left(e^{-t B_{T, N}} f\right)\right\|_{p} \leq C_{p}\|f\|_{p} & \left\|\sqrt{t} \operatorname{curl}\left(e^{-t B_{T, N}} f\right)\right\|_{p} \leq C_{p}^{\prime}\|f\|_{p} \\
\left\|t \nabla \operatorname{div}\left(e^{-t B_{T, N}} f\right)\right\|_{p} \leq K_{p}\|f\|_{p} & \left\|t \operatorname{curl} \operatorname{curl}\left(e^{-t B_{T, N}} f\right)\right\|_{p} \leq K_{p}^{\prime}\|f\|_{p}
\end{array}
$$

for all $f \in L^{p}\left(\Omega ; \mathbb{R}^{3}\right)$.

Proof. The estimates (3.16) and (3.17) in the corollary above come from the fact that for $p \in\left(q_{0}^{\prime}, q_{0}\right)$, the negative generators $B_{T, N}^{p}$ of the semigroups $\left(e^{-t B_{T, N}}\right)_{t \geq 0}$ satisfy

$$
\begin{aligned}
\mathrm{D}\left(B_{T, N}^{p}\right)= & \left\{u \in L^{p}\left(\Omega ; \mathbb{R}^{3}\right) ; \operatorname{div} u \in W^{1, p}\left(\Omega ; \mathbb{R}^{3}\right), \operatorname{curl} u \in L^{p}\left(\Omega ; \mathbb{R}^{3}\right),\right. \\
& \left.\quad \operatorname{curl} \operatorname{curl} u \in L^{p}\left(\Omega ; \mathbb{R}^{3}\right), \nu \cdot u=0 \text { and } \nu \times \operatorname{curl} u=0 \text { on } \partial \Omega\right\} \\
B_{T, N}^{p} u= & -\Delta u, \quad u \in \mathrm{D}\left(B_{T, N}^{p}\right) .
\end{aligned}
$$

This can be proved the same way we proved Proposition 3.5, (case $p=2$ ) using the fact that $\mathbb{P}$ and $\mathbb{P}_{N}$ are bounded in $L^{p}(\Omega ; \mathbb{R})$.

Remark 3.10. Let $w \in L^{2}\left(\Omega ; \mathbb{R}^{3}\right)$ such that $\operatorname{curl} w \in L^{2}\left(\Omega ; \mathbb{R}^{3}\right)$ and $\nu \times w=0$ on $\partial \Omega$. Then $\nu \cdot \operatorname{curl} w=0$ in $H^{-\frac{1}{2}}(\partial \Omega)$.

If the operator $B_{T}$ is restriced on $H_{D}$ and the operator $B_{N}$ on $H_{N}$, the following HodgeStokes operators $A_{T}$ and $A_{N}$ defined by

$$
\begin{aligned}
\mathrm{D}\left(A_{T}\right) & =\left\{u \in H_{D} \cap W_{T} ; \operatorname{curl} \operatorname{curl} u \in L^{2}\left(\Omega ; \mathbb{R}^{3}\right) \text { and } \nu \times \operatorname{curl} u=0 \text { on } \partial \Omega\right\} \\
A_{T} u & =\operatorname{curl} \operatorname{curl} u \quad \text { for } u \in \mathrm{D}\left(A_{T}\right)
\end{aligned}
$$

and

$$
\mathrm{D}\left(A_{N}\right)=\left\{u \in H_{N} \cap W_{N} ; \operatorname{curl} \operatorname{curl} u \in L^{2}\left(\Omega ; \mathbb{R}^{3}\right)\right\}, \quad A_{N} u=\operatorname{curl} \operatorname{curl} u \quad \text { for } u \in \mathrm{D}\left(A_{N}\right)
$$

are obtained. Remark 3.10 ensures that if $u \in \mathrm{D}\left(A_{T}\right)$ as defined above, $\nu \cdot \operatorname{curl} \operatorname{curl} u=0$ on $\partial \Omega$, so that curl curl $u \in H_{D}$.

The properties (3.13) and (3.14), together with a duality argument and the fact that the projections $\mathbb{P}$ and $\mathbb{P}_{N}$ are bounded on $L^{p}\left(\Omega ; \mathbb{R}^{3}\right)$ for $p \in\left((3+\varepsilon)^{\prime}, 3+\varepsilon\right)$ prove that $\left(e^{-t A_{T}}\right)_{t \geq 0}$ extends to an analytic semigroup on $H_{D}^{p}$ (its generator is denoted by $-A_{T, p}$ ) and $\left(e^{-t \bar{A}_{N}}\right)_{t \geq 0}$ extends to an analytic semigroup on $H_{N}^{p}$ (its generator is denoted by $-A_{N, p}$ ) for all $p \in\left[\frac{6}{5}, q_{0}\right)$. Moreover, the estimates (3.16) and (3.17) are valid if $B_{T, N}$ is replaced by $A_{T, N}$ for all $p \in\left[\frac{6}{5}, q_{0}\right)$.

Lemma 3.11. If $u \in H_{D}^{3}$ and $\operatorname{curl} u \in L^{3}\left(\Omega ; \mathbb{R}^{3}\right)$, then $u \in H_{D}^{p}$ for all $p \in\left[3, q_{0}\right)$.

Proof. Thanks to the relation (3.9),

$$
u=\mathbb{P} u=\mathbb{P}\left(R_{2} \operatorname{curl} u+K_{1} u\right)
$$

since $\mathbb{P} \nabla R_{1} u=0$. The mapping properties of $R_{2}$ and $K_{1}$ show that $R_{2}$ curl $u+K_{1} u \in$ $L^{3}\left(\Omega, \mathbb{R}^{3}\right) \cap L^{6}\left(\Omega, \mathbb{R}^{3}\right)$, which proves the claim of the Lemma. This has been done in, e.g., [34, Sections 3 and 4]. 
Remark 3.12. One can actually prove that the operator $-A_{T, p}$ generates an analytic semigroup in $H_{D}^{p}$ for all $p \in(1,3+\varepsilon)$. The same holds for $-A_{N, p}$ on $H_{N}^{p}$. See [29] for more details.

Remark 3.13. In [50], M.E. Taylor conjectured that the Dirichlet-Stokes operator generates an analytic semigroup in $H_{D}^{p}$ for $p \in\left((3+\varepsilon)^{\prime}, 3+\varepsilon\right)$, which was proved in [48]. The question of optimality of this range is still open, the counterexample provided by P. Deuring in [14] is for $p>6$. We see here that, for the Hodge-Stokes operator, one can allow all $p \in(1,3+\varepsilon)$.

\subsection{The nonlinear Hodge-Navier-Stokes equations}

The nonlinear Hodge-Navier-Stokes system ((NS'), (Hbc))

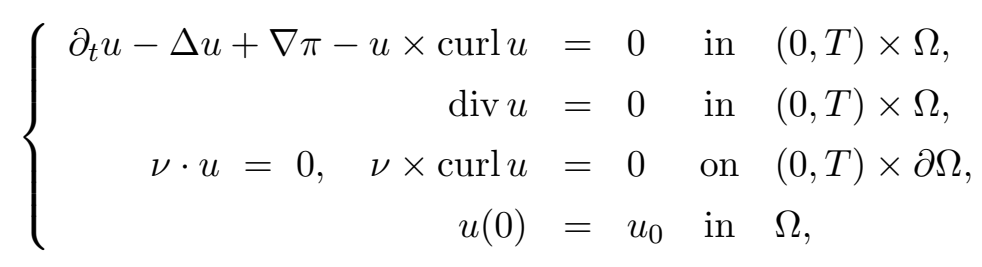

is considered for initial data $u_{0}$ in the critical space $H_{D}^{3}$ in the abstract form

$$
u^{\prime}(t)+A_{T, p} u(t)-\mathbb{P}(u(t) \times \operatorname{curl} u(t))=0, \quad u_{0} \in H_{D}^{3} .
$$

The idea to solve (3.19) is to apply the same method as in Sections 1 and 2.

With the properties of the Hodge-Stokes semigroup listed in the previous subsection (and more particularly Lemma 3.11), the following existence result for (3.19) is almost immediate. For $T \in(0, \infty]$, define the space $\mathscr{G}_{T}$ by

$$
\begin{aligned}
\mathscr{G}_{T}= & \left\{u \in \mathscr{C}_{b}\left([0, T) ; H_{D}^{3}\right) \cap \mathscr{C}\left((0, T) ; H_{D}^{3(1+\delta)}\right) ; \operatorname{curl} u \in \mathscr{C}\left((0, T) ; L^{3}\left(\Omega, \mathbb{R}^{3}\right)\right)\right. \\
& \text { with } \left.\sup _{0<s<T}\left(\left\|s^{\frac{\delta}{2(1+\delta)}} u(s)\right\|_{3(1+\delta)}+\|\sqrt{s} \operatorname{curl} u(s)\|_{3}\right)<\infty\right\}
\end{aligned}
$$

endowed with the norm

$$
\|u\|_{\mathscr{G}_{T}}=\sup _{0<s<T}\left(\|u(s)\|_{3}+\left\|s^{\frac{\delta}{2(1+\delta)}} u(s)\right\|_{3(1+\delta)}+\|\sqrt{s} \operatorname{curl} u(s)\|_{3}\right),
$$

where $0<\delta<\frac{\varepsilon}{3}(\varepsilon>0$ coming from (3.15)).

Theorem 3.14. Let $\Omega \subset \mathbb{R}^{3}$ be a bounded Lipschitz domain and let $u_{0} \in H_{D}^{3}$. Let $\gamma$ and $\Phi$ be defined by

$$
\gamma(t)=e^{-t A_{T, p}} u_{0}, \quad t \geq 0,
$$

and for $u, v \in \mathscr{G}_{T}$, and $t \in(0, T)$,

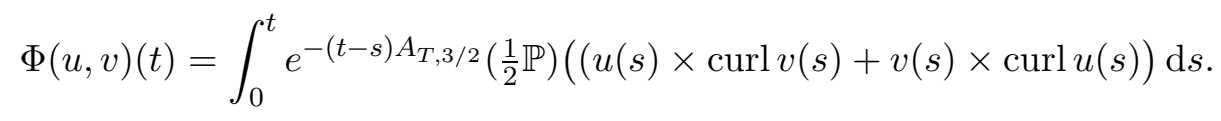

(i) If $\left\|u_{0}\right\|_{3}$ is small enough, then there exists a unique $u \in \mathscr{G}_{\infty}$ solution of $u=\gamma+\Phi(u, u)$.

(ii) For all $u_{0} \in H_{D}^{3}$, there exists $T>0$ and a unique $u \in \mathscr{G}_{T}$ solution of $u=\gamma+\Phi(u, u)$.

For a complete proof of this theorem, we refer to [34, Section 5]. 


\section{Robin boundary conditions}

As studied in [5], the system $\left(\left(\mathrm{NS}^{\prime}\right),(\mathrm{Rbc})\right)$ can also be considered. Recently, this has also been investigated in an $L^{2}$-setting for smooth domains $\Omega$ but with the friction coefficient $\alpha$ replaced by a (time-dependent) matrix $[0, T] \times \partial \Omega \ni(t, x) \mapsto \beta(t, x) \in \mathscr{M}_{3}(\mathbb{R})$ with $L_{t, x}^{\infty}$ coefficients, admitting $\nu(x)$ as eigenvector for almost every $(t, x)$; see [41]. It is also worth mentioning that the material here is part of a project with Jürgen Saal [42]. In the following, consider $\alpha \geq 0$ a constant. Note that the proofs in this section go through if $\alpha: \partial \Omega \rightarrow[0, \infty)$ is an $L^{\infty}$-function.

\subsection{The Robin-Hodge-Laplacian}

Recall the notations at the beginning of Subsection 3.1: $H=L^{2}\left(\Omega ; \mathbb{R}^{3}\right)$ and

$$
W_{T}:=\left\{u \in H ; \operatorname{curl} u \in H, \operatorname{div} u \in L^{2}(\Omega ; \mathbb{R}) \text { and } \nu \cdot u=0 \text { on } \partial \Omega\right\} .
$$

On $W_{T} \times W_{T}$, define the form

$$
b_{\alpha}: W_{T} \times W_{T} \rightarrow \mathbb{R}, \quad b_{\alpha}(u, v)=\langle\operatorname{curl} u, \operatorname{curl} v\rangle_{\Omega}+\langle\operatorname{div} u, \operatorname{div} v\rangle_{\Omega}+\langle\alpha u, v\rangle_{\partial \Omega} .
$$

Recall that according to (3.3), any $u \in W_{T}$ admits an $L^{2}$-trace on $\partial \Omega$, so that $\langle\alpha u, v\rangle_{\partial \Omega}$ makes sense for every $u, v \in W_{T}$.

Remark 4.1. The previous property holds also in $L^{p}, 1<p<\infty$, provided $\Omega$ is of class $\mathscr{C}^{1}$. More precisely, any $u \in L^{p}\left(\Omega, \mathbb{R}^{3}\right)$ with $\operatorname{curl} u \in L^{p}\left(\Omega, \mathbb{R}^{3}\right)$, div $u \in L^{p}(\Omega, \mathbb{R})$ and $\nu \cdot u=0$ on $\partial \Omega$ admits an $L^{p}$-trace on $\partial \Omega$ which satisfies

$$
\left\|u_{\left.\right|_{\partial \Omega}}\right\|_{L^{p}\left(\partial \Omega ; \mathbb{R}^{3}\right)} \leq C\left(\|u\|_{p}+\|\operatorname{curl} u\|_{p}+\|\operatorname{div} u\|_{p}\right) .
$$

See, e.g., [32, Proposition 6.2]: in the case of a $\mathscr{C}^{1}$ domain $\Omega$, the exponent $q_{\Omega}$ in that result (related to the solvability of the Poisson problem for Neumann boundary data and the regularity of the Poisson problem for Dirichlet boundary data) is equal to $\infty$.

The form $b_{\alpha}$ is continuous, bilinear, symmetric, coercive and sectorial, so that the associated operator $B_{\alpha}$ on $H$ is self-adjoint, $-B_{\alpha}$ generates an analytic semigroup of contractions and $D\left(B_{\alpha}^{1 / 2}\right)=W_{T}$. The operator $B_{\alpha}$ is called the Hodge-Robin-Laplacian. It has the following description:

$$
\begin{aligned}
\mathrm{D}\left(B_{\alpha}\right) & =\left\{u \in W_{T} ; \nabla \operatorname{div} u \in H, \operatorname{curl} \operatorname{curl} u \in H \text { and } \nu \times \operatorname{curl} u=\alpha u \text { on } \partial \Omega\right\} \\
B_{\alpha} u & =-\Delta u, \quad u \in \mathrm{D}\left(B_{\alpha}\right) .
\end{aligned}
$$

Remark that for $u \in W_{T}, u_{\left.\right|_{\partial \Omega}} \in L^{2}\left(\partial \Omega ; \mathbb{R}^{3}\right)$ and if moreover curl curl $u \in H$, the tangential vector field $\nu \times \operatorname{curl} u$ belongs to $H^{-\frac{1}{2}}\left(\partial \Omega ; \mathbb{R}^{3}\right)$. Therefore, the identity $\nu \times \operatorname{curl} u=\alpha u$ above holds in $H^{-\frac{1}{2}}\left(\partial \Omega ; \mathbb{R}^{3}\right)$. The proof of (4.1) follows the lines of the proof of Proposition 3.5, thanks to the following result (see, e.g., [41, Lemma 2.3], inspired by [32, Proof of Proposition 2.4 (iii)]) of which we also give the proof.

Lemma 4.2. 1. Let $g \in L^{2}\left(\partial \Omega, \mathbb{R}^{3}\right)$. Then there exists $w \in H$ with $\operatorname{curl} w \in H$ such that for all $\phi \in W_{T}$

$$
\langle g, \phi\rangle_{\partial \Omega}=\langle\operatorname{curl} w, \phi\rangle_{\Omega}-\langle w, \operatorname{curl} \phi\rangle_{\Omega} .
$$

Moreover, there exists $C>0$ such that

$$
\|w\|_{H}+\|\operatorname{curl} w\|_{H} \leq C\|g\|_{L^{2}\left(\partial \Omega, \mathbb{R}^{3}\right)} .
$$


2. If in addition $g \in L_{\mathrm{tan}}^{2}\left(\partial \Omega ; \mathbb{R}^{3}\right)$ (which means that $g \in L^{2}\left(\partial \Omega ; \mathbb{R}^{3}\right)$ and $\nu \cdot g=0$ on $\partial \Omega)$, then there exists $w \in H$ such that $\operatorname{curl} w \in H$ and (4.2) holds for all $\phi \in H^{1}(\Omega)$. And in that case $g=\nu \times w$ in $H^{-1 / 2}\left(\partial \Omega ; \mathbb{R}^{3}\right)$.

Proof. 1. Define the space $X:=\left\{(\phi, \operatorname{curl} \phi) ; \phi \in W_{T}\right\}$. It is a closed subspace of $H \times H$. As already mentioned, every $\phi \in W_{T}$ admits an $L^{2}$-trace at the boundary $\partial \Omega$ and therefore $\nu \times \phi \in L^{2}\left(\partial \Omega ; \mathbb{R}^{3}\right)$ for all $\phi \in W_{T}$. Since $g \in L^{2}\left(\partial \Omega ; \mathbb{R}^{3}\right)$, it is immediate that $\nu \times g \in$ $L^{2}\left(\partial \Omega ; \mathbb{R}^{3}\right)=\left(L^{2}\left(\partial \Omega ; \mathbb{R}^{3}\right)\right)^{\prime}$. Thus, $\nu \times g$ acts as a linear functional on $X$ as follows:

$$
(\nu \times g)(\phi, \operatorname{curl} \phi):=\langle\nu \times g, \nu \times \phi\rangle_{\partial \Omega} \quad \text { for all } \phi \in W_{T} .
$$

By the Hahn-Banach theorem, there exist $\left(v_{1}, v_{2}\right) \in H \times H$ such that

$$
(\nu \times g)(\phi, \operatorname{curl} \phi)=\left\langle v_{1}, \operatorname{curl} \phi\right\rangle_{\Omega}+\left\langle v_{2}, \phi\right\rangle_{\Omega} \quad \text { for all } \phi \in W_{T},
$$

where $(H \times H)^{\prime}$ has been identified with $H \times H$. Choose $\phi \in H_{0}^{1}\left(\Omega ; \mathbb{R}^{3}\right) \subset W_{T}$ and obtain that

$$
0=H^{-1}\left\langle\operatorname{curl} v_{1}+v_{2}, \phi\right\rangle_{H_{0}^{1}} .
$$

This gives that $\operatorname{curl} v_{1}+v_{2}=0$ in $H^{-1}\left(\Omega ; \mathbb{R}^{3}\right)$. Set $w:=-v_{1} \in H$, so that $\operatorname{curl} w=v_{2} \in H$. Moreover,

$$
\langle\nu \times g, \nu \times \phi\rangle_{\partial \Omega}=-\langle w, \operatorname{curl} \phi\rangle_{\Omega}+\langle\operatorname{curl} w, \phi\rangle_{\Omega} \quad \text { for all } \phi \in W_{T} .
$$

Since $\phi \in W_{T}, \phi_{\mid \partial \Omega} \in L_{\tan }^{2}\left(\partial \Omega, \mathbb{R}^{3}\right)$ and it is clear that $\phi=(\nu \times \phi) \times \nu$, so that the left-hand side of (4.4) coincides with

$$
\langle g, \phi\rangle_{\partial \Omega} \quad \text { for all } \phi \in W_{T},
$$

which proves (4.2).

The existence of $C>0$ such that (4.3) holds follows from the Closed Graph Theorem since $\{u \in H ; \operatorname{curl} u \in H\}$ is complete for the norm $\|u\|_{2}+\|\operatorname{curl} u\|_{2}$.

2. Assume now that $g \in L_{\text {tan }}^{2}\left(\partial \Omega ; \mathbb{R}^{3}\right)$. Let $w \in H$ such that curl $w \in H$ and (4.2) holds. Since $\nu \times g \in L^{2}\left(\partial \Omega ; \mathbb{R}^{3}\right)$, we can approach it in $L^{2}\left(\partial \Omega ; \mathbb{R}^{3}\right)$ by a sequence $\left(\varphi_{n}\right)_{n \in \mathbb{N}}$ of vector fields $\varphi_{n} \in H^{1 / 2}\left(\partial \Omega, \mathbb{R}^{3}\right)$. In particular,

$$
\varphi_{n} \times \nu \longrightarrow(\nu \times g) \times \nu=g \quad \text { in } L^{2}\left(\partial \Omega ; \mathbb{R}^{3}\right) \text { as } n \rightarrow \infty .
$$

By assertion 1, for each $n \in \mathbb{N}$ there exists $w_{n} \in H$ such that curl $w_{n} \in H$ satisfying

$$
\left\langle\varphi_{n} \times \nu, \phi\right\rangle_{\partial \Omega}=\left\langle\operatorname{curl} w_{n}, \phi\right\rangle_{\Omega}-\left\langle w_{n}, \operatorname{curl} \phi\right\rangle_{\Omega} \quad \text { for all } \phi \in W_{T} .
$$

Thanks to the estimate (4.3), it is immediate that

$$
w_{n} \underset{n \rightarrow \infty}{\longrightarrow} w \quad \text { and } \quad \operatorname{curl} w_{n} \underset{n \rightarrow \infty}{\longrightarrow} \operatorname{curl} w \quad \text { in } H .
$$

Let now $\phi \in H^{1}\left(\Omega ; \mathbb{R}^{3}\right)$. For $\varepsilon>0$, let $\phi_{\varepsilon}=\left(1+\varepsilon B_{T}\right)^{-1} \phi$. Then $\phi_{\varepsilon} \in W_{T}$ and thanks to Lemma 3.6,

$$
\phi_{\varepsilon} \underset{\varepsilon \rightarrow 0}{\longrightarrow} \phi \quad \text { and } \quad \operatorname{curl} \phi_{\varepsilon}=\left(1+\varepsilon B_{N}\right)^{-1} \operatorname{curl} \phi \underset{\varepsilon \rightarrow 0}{\longrightarrow} \operatorname{curl} \phi \quad \text { in } H .
$$

This implies also that

$$
\nu \times \phi_{\varepsilon} \underset{\varepsilon \rightarrow 0}{\longrightarrow} \nu \times \phi \quad \text { in } H^{-1 / 2}\left(\partial \Omega ; \mathbb{R}^{3}\right)
$$


Therefore, for all $\varepsilon>0$ and $n \in \mathbb{N}$

$$
\left\langle\nu \times \phi_{\varepsilon}, \varphi_{n}\right\rangle_{\partial \Omega}=\left\langle\varphi_{n} \times \nu, \phi_{\varepsilon}\right\rangle_{\partial \Omega}=\left\langle\operatorname{curl} w_{n}, \phi_{\varepsilon}\right\rangle_{\Omega}-\left\langle w_{n}, \operatorname{curl} \phi_{\varepsilon}\right\rangle_{\Omega} .
$$

First take the limit as $\varepsilon$ goes to 0 and obtain (recall that $\varphi_{n} \in H^{1 / 2}\left(\partial \Omega ; \mathbb{R}^{3}\right)$ )

$$
H^{-1 / 2}\left\langle\nu \times \phi, \varphi_{n}\right\rangle_{H^{1 / 2}}=\left\langle\operatorname{curl} w_{n}, \phi\right\rangle_{\Omega}-\left\langle w_{n}, \operatorname{curl} \phi\right\rangle_{\Omega} .
$$

Since $\phi \in H^{1}\left(\Omega, \mathbb{R}^{3}\right)$, the first term of the latter equation is also equal to $\left\langle\varphi_{n} \times \nu, \phi\right\rangle_{\partial \Omega}$. Taking the limit as $n$ goes to $\infty$ yields

$$
\langle g, \phi\rangle_{\partial \Omega}=\langle\operatorname{curl} w, \phi\rangle_{\Omega}-\langle w, \operatorname{curl} \phi\rangle_{\Omega}
$$

which proves the claim made in 2.

Remark 4.3. If $\Omega$ is of class $\mathscr{C}^{1}$, one can prove that Lemma 4.2 is also valid in $L^{p}$ instead of $L^{2}$ for all $p \in(1, \infty)$, identifying the dual of $L^{p}$ with $L^{p^{\prime}}$ (noting that $q_{0}$ defined in Proposition 3.8 is equal to $\infty$ ).

Proof of (4.1). For the time being, denote by $D_{\alpha}$ the set on the right-hand side of (4.1). Let $u \in D_{\alpha}: \Delta u=-\operatorname{curl} \operatorname{curl} u+\nabla \operatorname{div} u \in H$ and for all $v \in W_{T} \cap H^{1}\left(\Omega ; \mathbb{R}^{3}\right)$,

$$
\begin{aligned}
\langle-\Delta u, v\rangle_{\Omega} & =\langle\operatorname{curl} \operatorname{curl} u, v\rangle_{\Omega}-\langle\nabla \operatorname{div} u, v\rangle_{\Omega} \\
& =\langle\operatorname{curl} u, \operatorname{curl} v\rangle_{\Omega}+\langle\nu \times \operatorname{curl} u, v\rangle_{\partial \Omega}+\langle\operatorname{div} u, \operatorname{div} v\rangle_{\Omega} \\
& =\langle\operatorname{curl} u, \operatorname{curl} v\rangle_{\Omega}+\langle\operatorname{div} u, \operatorname{div} v\rangle_{\Omega}+\alpha\langle u, v\rangle_{\partial \Omega} \\
& =b_{\alpha}(u, v) .
\end{aligned}
$$

The second equality comes from the integration by parts formula. In the third equality the characterization of elements in $D_{\alpha}$ has been used. Thanks to the density of $W_{T} \cap H^{1}\left(\Omega ; \mathbb{R}^{3}\right)$ in $W_{T}$, this proves the inclusion $D_{\alpha} \subseteq D\left(B_{\alpha}\right)$ and that $B_{\alpha} u=-\Delta u$ for $u \in D_{\alpha}$.

Conversely, let $u \in D\left(B_{\alpha}\right)$. Let $\eta=-B_{\alpha} u \in H, g=\alpha u$. Since $u_{\mid \partial \Omega} \in L_{\tan }^{2}\left(\partial \Omega ; \mathbb{R}^{3}\right)$, Lemma 4.2 shows the existence of $w \in H$ with $\operatorname{curl} w \in H$ such that $\alpha u=\nu \times w$ on $\partial \Omega$. Therefore, the boundary value $g=\alpha u$ satisfies the conditions of [32, Theorem 1.2] with $p=2$. Then there exists a unique $\tilde{u}$ satisfying

$$
\left\{\begin{array}{l}
\tilde{u} \in W_{T}, \operatorname{curl} \operatorname{curl} \tilde{u} \in H, \operatorname{div} \tilde{u} \in H^{1}(\Omega), \\
\Delta \tilde{u}=\eta \in H, \\
\nu \times \operatorname{curl} \tilde{u}=g \in H^{-1 / 2}\left(\partial \Omega ; \mathbb{R}^{3}\right)
\end{array}\right.
$$

For all $v \in W_{T}$, integrating by parts,

$$
\begin{aligned}
\langle\operatorname{curl} \tilde{u}, \operatorname{curl} v\rangle_{\Omega}+\langle\operatorname{div} \tilde{u}, \operatorname{div} v\rangle_{\Omega} & =\langle-\Delta \tilde{u}, v\rangle_{\Omega}-\langle\nu \times \operatorname{curl} \tilde{u}, v\rangle_{\partial \Omega} \\
& =\langle-\eta, v\rangle_{\Omega}-\langle g, v\rangle_{\partial \Omega} \\
& =\left\langle B_{\alpha} u, v\right\rangle_{\Omega}-\langle\alpha u, v\rangle_{\partial \Omega} \\
& =b_{\alpha}(u, v)-\alpha\langle u, v\rangle_{\partial \Omega} \\
& =\langle\operatorname{curl} u, \operatorname{curl} v\rangle_{\Omega}+\langle\operatorname{div} u, \operatorname{div} v\rangle_{\Omega} .
\end{aligned}
$$

The second equality comes from the fact that $\tilde{u}$ is the solution of (4.6). The third equality is a simple reformulation of the previous line using the notations introduced before. The fourth equality uses the fact that $B_{\alpha}$ is the operator associated with the form $b_{\alpha}$. Finally, the last equality comes directly from the definition of $b_{\alpha}$. Therefore, we proved that $v=u-\tilde{u} \in W_{T}$ and satisfies $\operatorname{curl} v=0$ and $\operatorname{div} v=0$. Since $\Omega$ is simply connected, this proves that $v=0$, or equivalently $u=\tilde{u}$, and then that $u \in D_{\alpha}$ from which follows the inclusion $D\left(B_{\alpha}\right) \subseteq D_{\alpha}$.

Ultimately, it has been proved that $D\left(B_{\alpha}\right)=D_{\alpha}$. 
As in the case of Proposition 3.7, Gaffney-type estimates hold:

Proposition 4.4. There exist two constants $C, c>0$ such that for any open sets $E, F \subset \mathbb{R}^{3}$ such that $\operatorname{dist}(E, F)>0$ and for all $t>0, f \in H$ and

$$
u=\left(\operatorname{Id}+t^{2} B_{\alpha}\right)^{-1}\left(1_{F} f\right),
$$

it holds

$$
\left\|1_{E} u\right\|_{2}+t\left\|1_{E} \operatorname{div} u\right\|_{2}+t\left\|1_{E} \operatorname{curl} u\right\|_{2}+t \sqrt{\alpha}\left\|1_{E} u\right\|_{L^{2}\left(\partial \Omega ; \mathbb{R}^{3}\right)} \leq C e^{-\frac{\operatorname{dist}(E, F)}{t}}\left\|1_{F} f\right\|_{2} .
$$

Proof. The proof goes as in the case $\alpha=0$ (Proposition 3.7 for $B_{T}$ ). Choose a smooth cut-off function $\xi: \mathbb{R}^{3} \rightarrow \mathbb{R}$ satisfying $\xi=1$ on $E, \xi=0$ on $F$ and $\|\nabla \xi\|_{\infty} \leq \frac{k}{\operatorname{dist}(E, F)}$. Then define $\eta=e^{\delta \xi}$ where $\delta>0$ is to be chosen later. Next, take the scalar product of the equation

$$
u-t^{2} \Delta u=1_{F} f, \quad u \in \mathrm{D}\left(B_{\alpha}\right)
$$

with the function $v=\eta^{2} u$. Since $\eta=1$ on $F$ and $\|u\|_{2} \leq\left\|1_{F} f\right\|_{2}$, it is easy to check then that

$$
\begin{aligned}
& \|\eta u\|_{2}^{2}+t^{2}\|\eta \operatorname{div} u\|_{2}^{2}+t^{2}\|\eta \operatorname{curl} u\|_{2}^{2}+t^{2} \alpha\|\eta u\|_{L^{2}\left(\partial \Omega ; \mathbb{R}^{3}\right)}^{2} \\
\leq & \left\|1_{F} f\right\|_{2}^{2}+2 \alpha\|\nabla \xi\|_{\infty} t^{2}\|\eta u\|_{2}\left(\|\eta \operatorname{div} u\|_{2}+\|\eta \operatorname{curl} u\|_{2}\right)
\end{aligned}
$$

and therefore, using the estimate on $\|\nabla \xi\|_{\infty}$ and choosing $\delta=\frac{\operatorname{dist}(E, F)}{4 k t}$,

$$
\|\eta u\|_{2}^{2}+t^{2}\|\eta \operatorname{div} u\|_{2}^{2}+t^{2}\|\eta \operatorname{curl} u\|_{2}^{2}+t^{2} \alpha\|\eta u\|_{L^{2}\left(\partial \Omega ; \mathbb{R}^{3}\right)}^{2} \leq 2\left\|1_{F} f\right\|_{2}^{2} .
$$

Using now the fact that $\eta=e^{\delta}$ on $E$,

$$
\left\|1_{E} u\right\|_{2}+t\left\|1_{E} \operatorname{div} u\right\|_{2}+t\left\|1_{E} \operatorname{curl} u\right\|_{2}+t \sqrt{\alpha}\left\|11_{E} u\right\|_{L^{2}\left(\partial \Omega ; \mathbb{R}^{3}\right)} \leq \sqrt{2} e^{-\frac{\operatorname{dist}(E, F)}{4 k t}}\left\|1_{F} f\right\|_{2},
$$

which gives (4.7) with $C=\sqrt{2}$ and $c=\frac{1}{4 k}$.

As before, with a slight modification of the proof, it can be shown that for all $\theta \in$ $(0, \pi)$ there exist two constants $C, c>0$ such that for any open sets $E, F \subset \mathbb{R}^{3}$ such that $\operatorname{dist}(E, F)>0$ and for all $z \in \Sigma_{\pi-\theta}=\{\omega \in \mathbb{C} \backslash\{0\} ;|\arg z|<\pi-\theta\}, f \in H$ and

$$
u=\left(z \operatorname{Id}+B_{\alpha}\right)^{-1}\left(\mathbb{1}_{F} f\right),
$$

it holds

$$
\begin{aligned}
|z|\left\|1_{E} u\right\|_{2} & +|z|^{\frac{1}{2}}\left\|1_{E} \operatorname{div} u\right\|_{2}+|z|^{\frac{1}{2}}\left\|1_{E} \operatorname{curl} u\right\|_{2} \\
& +|z|^{\frac{1}{2}} \sqrt{\alpha}\left\|1_{E} u\right\|_{L^{2}\left(\partial \Omega ; \mathbb{R}^{3}\right)} \leq C e^{-c \operatorname{dist}(E, F)|z|^{\frac{1}{2}}}\left\|1_{F} f\right\|_{2} .
\end{aligned}
$$

With the same arguments as for the Hodge-Laplacian, the analogue of Proposition 3.8 and Corollary 3.9 can be obtained, as well as (3.18) for $B_{\alpha}$ : for all $p \in\left(q_{0}^{\prime}, q_{0}\right)$,

$\left\{z\left(z \operatorname{Id}+B_{\alpha}\right)^{-1}, z \in \Sigma_{\pi-\theta}\right\}$ is uniformly bounded in $L^{p}\left(\Omega ; \mathbb{R}^{3}\right) ;$

$\left(e^{-t B_{\alpha}}\right)_{t \geq 0}$ extends to a bounded analytic semigroup on $L^{p}\left(\Omega ; \mathbb{R}^{3}\right)$;

$\left\|\sqrt{t} \operatorname{div}\left(e^{-t B_{\alpha}} f\right)\right\|_{p} \leq C_{p}\|f\|_{p}, \quad\left\|\sqrt{t} \operatorname{curl}\left(e^{-t B_{\alpha}} f\right)\right\|_{p} \leq C_{p}^{\prime}\|f\|_{p} ;$

$\left\|t \nabla \operatorname{div}\left(e^{-t B_{\alpha}} f\right)\right\|_{p} \leq K_{p}\|f\|_{p}, \quad\left\|t \operatorname{curl} \operatorname{curl}\left(e^{-t B_{\alpha}} f\right)\right\|_{p} \leq K_{p}^{\prime}\|f\|_{p}$. 
Moreover, if $\Omega$ is of class $\mathscr{C}^{1}$, the following description of $B_{\alpha, p}$, the negative generator of $\left(e^{-t B_{\alpha}}\right)_{t \geq 0}$ in $L^{p}\left(\Omega ; \mathbb{R}^{3}\right)$ holds:

$$
\begin{aligned}
& \mathrm{D}\left(B_{\alpha, p}\right)=\left\{u \in L^{p}\left(\Omega ; \mathbb{R}^{3}\right) ; \operatorname{div} u \in W^{1, p}\left(\Omega ; \mathbb{R}^{3}\right), \operatorname{curl} u \in L^{p}\left(\Omega ; \mathbb{R}^{3}\right),\right. \\
& \left.\quad \operatorname{curl} \operatorname{curl} u \in L^{p}\left(\Omega ; \mathbb{R}^{3}\right), \nu \cdot u=0 \text { and } \nu \times \operatorname{curl} u=\alpha u \text { on } \partial \Omega\right\} \\
& B_{\alpha, p} u=-\Delta u, \quad u \in \mathrm{D}\left(B_{\alpha, p}\right),
\end{aligned}
$$

To prove that, the result in Remark 4.3 has been used, as well as the solvability of (4.6) in $L^{p}$ for $p$ in the interval $\left((3+\varepsilon)^{\prime}, 3+\varepsilon\right)=(1, \infty)$ in that case $([32$, Theorem 1.2] is also valid in this range of $p$ ).

\subsection{The Robin-Hodge-Stokes operator}

From now on, assume that $\Omega$ is of class $\mathscr{C}^{1}$. Let $p \in(1, \infty)$. Let $g \in L^{p}\left(\Omega ; \mathbb{R}^{3}\right)$, with $\operatorname{div} g=0$. By Remark 1.1 (also valid for $p \in(1, \infty)$ with the obvious changes), it holds $\nu \cdot g \in B_{p, p}^{-1 / p}(\partial \Omega)$ and also $\nu \cdot g$ satisfies the condition ${ }_{B_{p, p}^{-1 / p}(\partial \Omega)}\langle\nu \cdot g, 1\rangle_{B_{p^{\prime}, p^{\prime}}^{1 / p}(\partial \Omega)}=0$. By [19, Corollary 9.3], the problem

$$
q \in W^{1, p}(\Omega), \quad \Delta q=0 \text { in } \Omega, \quad \partial_{\nu} q=\nu \cdot g \text { on } \partial \Omega
$$

has a unique (modulo constants) solution satisfying moreover

$$
\|\nabla q\|_{p} \lesssim\|\nu \cdot g\|_{B_{p, p}^{-1 / p}(\partial \Omega)} \cdot
$$

Consider the operator

$$
\Gamma_{p}: \mathrm{D}\left(B_{\alpha, p}\right) \longrightarrow W^{1, p}(\Omega), \quad u \longmapsto q
$$

where $q$ is the solution of (4.14) with $g=-\operatorname{curl} \operatorname{curl} u$.

Lemma 4.5. For $p \in(1, \infty), u \in \mathrm{D}\left(B_{\alpha, p}\right)$, the following estimate holds

$$
\left\|\nabla \Gamma_{p} u\right\|_{p} \lesssim \alpha\left(\|\operatorname{curl} u\|_{p}+\|\operatorname{div} u\|_{p}\right) .
$$

Proof. Let $p \in(1, \infty)$ and $u \in \mathrm{D}\left(B_{\alpha, p}\right)$. Let $\varphi \in B_{p^{\prime}, p^{\prime}}^{1 / p}(\partial \Omega)$. Let $\Phi \in W^{1, p^{\prime}}(\Omega)$, so that $\Phi_{\mid \partial \Omega}=\varphi$ (recall that $\left.\frac{1}{p}=1-\frac{1}{p^{\prime}}\right)$. Thanks to the description of $\mathrm{D}\left(B_{\alpha, p}\right)$ given by (4.13) and the formula (3.1) (also valid in $L^{p}$ ), there holds

$$
\begin{aligned}
B_{p, p}^{-1 / p}(\partial \Omega) & \langle\nu \cdot \operatorname{curl} \operatorname{curl} u, \varphi\rangle_{B_{p^{\prime}, p^{\prime}}^{1 / p}(\partial \Omega)} \\
& =\langle\operatorname{curl} \operatorname{curl} u, \nabla \Phi\rangle_{\Omega}=\langle\nu \times \operatorname{curl} u, \nabla \Phi\rangle_{\partial \Omega} \\
& =\alpha\langle u, \nabla \Phi\rangle_{\partial \Omega}=\alpha\langle\operatorname{curl} w, \nabla \Phi\rangle_{\Omega},
\end{aligned}
$$

where $w \in L^{p}\left(\Omega ; \mathbb{R}^{3}\right)$ with $\operatorname{curl} w \in L^{p}\left(\Omega ; \mathbb{R}^{3}\right)$ is determined by Lemma 4.2, 2 (for $g=u$; see Remark 4.3). Therefore by Remark 4.1

$$
\|\nu \cdot \operatorname{curl} \operatorname{curl} u\|_{B_{p, p}^{-1 / p}(\partial \Omega)} \leq C\|\operatorname{curl} w\|_{p} \leq C\|u\|_{L^{p}\left(\partial \Omega ; \mathbb{R}^{3}\right)} \leq C\left(\|u\|_{p}+\|\operatorname{curl} u\|_{p}+\operatorname{div} u \|_{p}\right) .
$$

Since $\Omega$ is bounded, $\|u\|_{p}$ can be estimated in terms of $\|\operatorname{curl} u\|_{p}$ and $\|\operatorname{div} u\|_{p}$, which gives (4.16). 
Next result links the operator $\Gamma_{p}$ and $B_{\alpha, p}$ with the Robin-Hodge-Stokes resolvent problem for $z \in \Sigma_{\pi-\theta}$ :

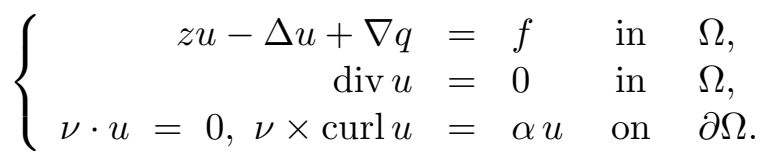

Proposition 4.6. Let $p \in(1, \infty)$. Let $z \in \Sigma_{\pi-\theta}$ and $f \in H_{D}^{p}$. Then $(u, q) \in \mathrm{D}\left(B_{\alpha, p}\right) \times$ $W^{1, p}(\Omega)$ is a solution of (4.17) if, and only if, $u \in \mathrm{D}\left(B_{\alpha, p}\right) \cap H_{D}^{p}$ satisfies $z u-\Delta u+\nabla \Gamma_{p} u=f$ and in that case $q=\Gamma_{p} u$.

Proof. $\Rightarrow$ : Assume that $(u, q) \in \mathrm{D}\left(B_{\alpha, p}\right) \times W^{1, p}(\Omega)$ is a solution of (4.17). Applying the divergence to the first equation of (4.17) and using the fact that $\operatorname{div} u=0$, there holds $\Delta \pi=0$. Moreover, taking the normal component at the boundary of the same equation, $\partial_{\nu} q=\nu \cdot \Delta u=-\nu \cdot \operatorname{curl} \operatorname{curl} u$ (recall that, since $f \in H_{D}^{p}, \nu \cdot f=0$ on $\partial \Omega$ ) and therefore $q$ satisfies (4.14) with $g=-\operatorname{curl} \operatorname{curl} u$, which implies by definition of $\Gamma_{p}$ that $q=\Gamma_{p} u$. This shows that $u \in \mathrm{D}\left(B_{\alpha, p}\right) \cap H_{D}^{p}$ and satisfies $z u-\Delta u+\nabla \Gamma_{p} u=f$.

$\Leftarrow$ : Conversely, let $u \in \mathrm{D}\left(B_{\alpha, p}\right) \cap H_{D}^{p}$ satisfying $z u-\Delta u+\nabla \Gamma_{p} u=f$ and define $v:=\operatorname{div} u \in$ $W^{1, p}(\Omega)$. Then $v$ satisfies $z v-\Delta v=0$ in $\Omega$ : apply the divergence to $z u-\Delta u+\nabla \Gamma_{p} u=$ $f$ and remark that $\operatorname{div} f=0$ and $\operatorname{div} \nabla \Gamma_{p} u=\Delta \Gamma_{p} u=0$. Moreover, taking the normal component of $z u-\Delta u+\nabla \Gamma_{p} u=f$ at the boundary, $-\partial_{\nu} v+\nu \cdot \operatorname{curl} \operatorname{curl} u+\partial_{\nu} \Gamma_{p} u=0$ on $\partial \Omega$ (we wrote $-\Delta u=-\nabla v+\operatorname{curl} \operatorname{curl} u$ ), and therefore $\partial_{\nu} v=0$ on $\partial \Omega$. Uniqueness of the Neumann problem for the Laplacian,

$$
\left(z v-\Delta v=0 \text { in } \Omega \quad \text { and } \quad \partial_{\nu} v=0 \text { on } \partial \Omega\right) \Longrightarrow(v=0),
$$

shows that $v=\operatorname{div} u=0$. Therefore, $\left(u, \Gamma_{p} u\right) \in \mathrm{D}\left(B_{\alpha, p}\right) \times W^{1, p}(\Omega)$ is a solution of (4.17).

Proposition 4.6 allows to define the part of $B_{\alpha, p}$ in $H_{D}^{p}$ as follows.

Definition 4.7. Let $p \in(1, \infty)$. The Robin-Hodge-Stokes operator denoted by $A_{\alpha, p}$ is an unbounded operator in $H_{D}^{p}$ defined by

$$
\mathrm{D}\left(A_{\alpha, p}\right)=\mathrm{D}\left(B_{\alpha, p}\right) \cap H_{D}^{p}, \quad A_{\alpha, p} u=-\Delta u+\nabla \Gamma_{p} u, \quad u \in \mathrm{D}\left(A_{\alpha, p}\right) .
$$

Remark 4.8. If $p=2$, it is easy to see that $A_{\alpha, 2}$ is the operator associated with the continuous, bilinear, symmetric, coercive form $a_{\alpha}$ defined as follows

$$
a_{\alpha}:\left(W_{T} \cap H_{D}\right) \times\left(W_{T} \cap H_{D}\right) \rightarrow \mathbb{R}, \quad a_{\alpha}(u, v):=\langle\operatorname{curl} u, \operatorname{curl} v\rangle_{\Omega}+\langle\alpha u, v\rangle_{\partial \Omega} .
$$

Therefore, $A_{\alpha, 2}$ is self adjoint and $-A_{\alpha, 2}$ is the generator of an analytic semigroup of contractions in $H_{D}$.

Lemma 4.9. Let $p \in[2, \infty)$ and $u \in \mathrm{D}\left(A_{\alpha, p}\right)$. Then $u \in L^{\frac{9 p}{4}}\left(\Omega ; \mathbb{R}^{3}\right)$.

Proof. By definition, if $u \in \mathrm{D}\left(A_{\alpha, p}\right)$, then $u$, $\operatorname{curl} u \in L^{p}\left(\Omega ; \mathbb{R}^{3}\right), \operatorname{div} u=0 \in L^{p}(\Omega)$ and $\nu \cdot u=0$ on $\partial \Omega$. By [31, Theorem 11.2] (note that $B_{p, p}^{1 / p} \hookrightarrow L^{\frac{3 p}{2}}$ in dimension 3), there holds $u \in L^{\frac{3 p}{2}}\left(\Omega ; \mathbb{R}^{3}\right)$. Apply the same reasoning to $\operatorname{curl} u$ : $\operatorname{curl} u, \operatorname{curl} \operatorname{curl} u \in L^{p}\left(\Omega ; \mathbb{R}^{3}\right)$, $\operatorname{div} \operatorname{curl} u=0 \in L^{p}(\Omega)$ and $\nu \times \operatorname{curl} u=\alpha u \in L^{p}\left(\partial \Omega ; \mathbb{R}^{3}\right)$, so that $\operatorname{curl} u \in L^{\frac{3 p}{2}}\left(\Omega ; \mathbb{R}^{3}\right)$. Using again that $\nu \cdot u=0$ on $\partial \Omega$, there holds $u \in L^{\frac{9 p}{4}}\left(\Omega ; \mathbb{R}^{3}\right)$. 
Theorem 4.10. For all $p \in(1, \infty)$, the operator $-A_{\alpha, p}$ generates an analytic semigroup in $H_{D}^{p}$ satisfying the estimates

$$
\left\|\sqrt{t} \operatorname{curl}\left(e^{-t A_{\alpha, p}} f\right)\right\|_{p} \leq C_{p}\|f\|_{p} \quad \text { and } \quad\left\|t \operatorname{curl} \operatorname{curl}\left(e^{-t A_{\alpha, p}} f\right)\right\|_{p} \leq K_{p}\|f\|_{p}
$$

for all $f \in H_{D}^{p}$ if $p \geq 2$.

Proof. Let $z \in \Sigma_{\pi-\theta}$. By Proposition 4.6,

$$
\left(z \operatorname{Id}+A_{\alpha, p}\right)=\left(\operatorname{Id}-\nabla \Gamma_{p}\left(z \operatorname{Id}+B_{\alpha, p}\right)^{-1}\right)\left(z \operatorname{Id}+B_{\alpha, p}\right) .
$$

Lemma 4.5 and (4.11) imply that for all $f \in L^{p}\left(\Omega ; \mathbb{R}^{3}\right)$,

$\left\|\nabla \Gamma_{p}\left(z \operatorname{Id}+B_{\alpha, p}\right)^{-1} f\right\|_{p} \lesssim \alpha\left(\left\|\operatorname{curl}\left(z \operatorname{Id}+B_{\alpha, p}\right)^{-1} f\right\|_{p}+\left\|\operatorname{div}\left(z \operatorname{Id}+B_{\alpha, p}\right)^{-1} f\right\|_{p}\right) \leq C \frac{\alpha}{\sqrt{|z|}}\|f\|_{p}$.

This proves that, for $|z|$ large enough $\left(|z| \geq 4 C^{2} \alpha^{2}\right), z \operatorname{Id}+A_{\alpha, p}: \mathrm{D}\left(A_{\alpha, p}\right) \rightarrow H_{D}^{p}$ is invertible with

$$
\left(z \operatorname{Id}+A_{\alpha, p}\right)^{-1}=\left(z \operatorname{Id}+B_{\alpha, p}\right)^{-1}\left(\operatorname{Id}-\nabla \Gamma_{p}\left(z \operatorname{Id}+B_{\alpha, p}\right)^{-1}\right)^{-1}
$$

and

$$
\left\|z\left(z \operatorname{Id}+A_{\alpha, p}\right)^{-1}\right\|_{\mathscr{L}\left(H_{D}^{p}\right)} \leq 2\left\|z\left(z \operatorname{Id}+B_{\alpha, p}\right)^{-1}\right\|_{\mathscr{L}\left(L^{p}\left(\Omega ; \mathbb{R}^{3}\right)\right)} \lesssim 1 .
$$

Moreover, the same reasoning gives

$$
\left\|\sqrt{|z|} \operatorname{curl}\left(z \operatorname{Id}+A_{\alpha, p}\right)^{-1}\right\|_{\mathscr{L}\left(H_{D}^{p} ; L^{p}\left(\Omega ; \mathbb{R}^{3}\right)\right)} \leq 2\left\|\sqrt{|z|} \operatorname{curl}\left(z \operatorname{Id}+B_{\alpha, p}\right)^{-1}\right\|_{\mathscr{L}\left(L^{p}\left(\Omega ; \mathbb{R}^{3}\right)\right)} \lesssim 1
$$

and

$$
\left\|\operatorname{curl} \operatorname{curl}\left(z \operatorname{Id}+A_{\alpha, p}\right)^{-1}\right\|_{\mathscr{L}\left(H_{D}^{p} ; L^{p}\left(\Omega ; \mathbb{R}^{3}\right)\right)} \leq 2\left\|\operatorname{curl} \operatorname{curl}\left(z \operatorname{Id}+B_{\alpha, p}\right)^{-1}\right\|_{\mathscr{L}\left(L^{p}\left(\Omega ; \mathbb{R}^{3}\right)\right)} \lesssim 1
$$

To prove that $z \operatorname{Id}+A_{\alpha, p}: \mathrm{D}\left(A_{\alpha, p}\right) \rightarrow H_{D}^{p}$ is invertible if $z \in \Sigma_{\pi-\theta}$ with $|z| \leq 4 C^{2} \alpha^{2}$, proceed by induction. The assertion is proved for $p \geq 2$ (the range is obtained $1<p \leq 2$ by duality since $A_{\alpha, 2}$ is self adjoint in $\left.H_{D}\right)$. Assume first that $p \in\left[2, \frac{9}{2}\right]$, so that $\mathrm{D}\left(A_{\alpha, 2}\right) \hookrightarrow H_{D}^{p}$ by Lemma 4.9. Let $z \in \Sigma_{\pi-\theta}$ with $|z| \leq 4 C^{2} \alpha^{2}$ and let $\omega=z+8 C^{2} \alpha^{2}$. There holds $\omega \in \Sigma_{\pi-\theta}$ and $|\omega| \geq 8 C^{2} \alpha^{2}-|z| \geq 4 C^{2} \alpha^{2}$. Therefore, for $f \in H_{D}^{p} \hookrightarrow H_{D}$,

$$
\left(z \operatorname{Id}+A_{\alpha, 2}\right)^{-1} f=\left(\omega \mathrm{Id}+A_{\alpha, p}\right)^{-1} f+8 C^{2} \alpha^{2}\left(\omega \mathrm{Id}+A_{\alpha, p}\right)^{-1}\left(z \operatorname{Id}+A_{\alpha, 2}\right)^{-1} f,
$$

which gives

$$
\left\|\left(z \mathrm{Id}+A_{\alpha, 2}\right)^{-1} f\right\|_{p} \leq C_{\alpha}\|f\|_{p}
$$

and this proves that $z \mathrm{Id}+A_{\alpha, p}: \mathrm{D}\left(A_{\alpha, p}\right) \rightarrow H_{D}^{p}$ is invertible with the norm of its inverse controlled by a constant depending on $\alpha$. For any $p \geq 2$, the previous procedure can be iterated using again Lemma 4.9 valid for all $p \geq 2$. Estimates of the form (4.20) and (4.21) are straightforward. Eventually, the result claimed in Theorem 4.10 is obtained for $p \geq 2$. As mentioned earlier, the case $1<p \leq 2$ is obtained by duality. 


\subsection{The nonlinear Robin-Hodge-Navier-Stokes equations}

The nonlinear Robin-Hodge-Navier-Stokes system ((NS'), (Rbc))

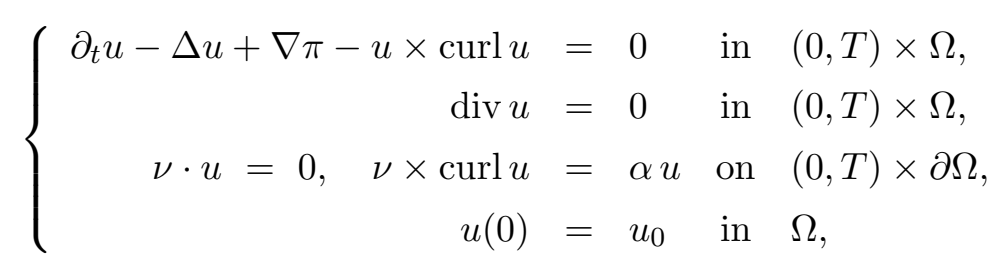

for initial data $u_{0}$ is considered in the critical space $H_{D}^{3}$ in the abstract form

$$
u^{\prime}(t)+A_{\alpha, p} u(t)-\mathbb{P}(u(t) \times \operatorname{curl} u(t))=0, \quad u_{0} \in H_{D}^{3} .
$$

Recall that $\mathscr{C}^{1}$ domains $\Omega$ are considered here. The idea to solve (4.22) is to apply the same method as in previous Sections.

With the properties of the Robin-Hodge-Stokes semigroup listed in particular in Theorem 4.10, the following existence result for (4.22) is almost immediate. For $T \in(0, \infty]$, define the space $\mathscr{H}_{T}$ by

$$
\begin{aligned}
\mathscr{H}_{T}= & \left\{u \in \mathscr{C}_{b}\left([0, T) ; H_{D}^{3}\right) ; \operatorname{curl} u \in \mathscr{C}\left((0, T) ; L^{3}\left(\Omega, \mathbb{R}^{3}\right)\right)\right. \\
& \text { with } \left.\sup _{0<s<T}\|\sqrt{s} \operatorname{curl} u(s)\|_{3}<\infty\right\}
\end{aligned}
$$

endowed with the norm

$$
\|u\|_{\mathscr{H}_{T}}=\sup _{0<s<T}\left(\|u(s)\|_{3}+\|\sqrt{s} \operatorname{curl} u(s)\|_{3}\right) .
$$

Theorem 4.11. Let $\Omega \subset \mathbb{R}^{3}$ be a bounded Lipschitz domain and let $u_{0} \in H_{D}^{3}$. Let $\gamma$ and $\Phi$ be defined by

$$
\gamma(t)=e^{-t A_{\alpha, 3}} u_{0}, \quad t \geq 0,
$$

and for $u, v \in \mathscr{H}_{T}$, and $t \in(0, T)$,

$$
\Phi(u, v)(t)=\int_{0}^{t} e^{-(t-s) A_{\alpha, 2}}\left(\frac{1}{2} \mathbb{P}\right)((u(s) \times \operatorname{curl} v(s)+v(s) \times \operatorname{curl} u(s)) \mathrm{d} s .
$$

(i) If $\left\|u_{0}\right\|_{3}$ is small enough, then there exists a unique $u \in \mathscr{H}_{\infty}$ solution of $u=\gamma+\Phi(u, u)$.

(ii) For all $u_{0} \in H_{D}^{3}$, there exists $T>0$ and a unique $u \in \mathscr{H}_{T}$ solution of $u=\gamma+\Phi(u, u)$.

Elements of the proof. Remark that, as in Lemma 3.11, for $u \in \mathscr{H}_{T}$, (thanks to (3.9)) there holds $u=\mathbb{P}\left(R_{2} \operatorname{curl} u+K_{1} u\right) \in \mathscr{C}\left((0, T) ; H_{D}^{6}\right)$ with $\sup _{0<s<T} \sqrt{s}\|u(s)\|_{6} \leq\|u\|_{\mathscr{H}_{T}}$. The proof goes as in the previous sections.

\section{Conclusion}

In the case of a smooth bounded domain in $\mathbb{R}^{n}$, it was proved by Y. Giga and T. Miyakawa in [22] that the Dirichlet-Navier-Stokes system admits a local mild solution for initial values in $L^{n}$ (critical space for the system in dimension $n$ ). Their method relies on the fact that 
the Dirichlet-Stokes operator, as defined in Section 1, extends to all $L^{p}$ spaces and is the negative generator of an analytic semigroup there, which was proved in [21]. The situation in Lipschitz domains is different. For instance, P. Deuring provided in [14] an example of a domain with one conical singularity such that the Dirichlet-Stokes semigroup does not extend to an analytic semigroup in $L^{p}$ for $p$ large, away from 2 (in this example, $p>6$ ).

As already mentioned, E. Fabes, O. Mendez and M. Mitrea proved in [19] that the orthogonal projection $\mathbb{P}$ defined in Section 1 on $L^{2}\left(\Omega ; \mathbb{R}^{3}\right)$ extends to a bounded projection on $L^{p}\left(\Omega ; \mathbb{R}^{3}\right)$ for $p$ in an open interval containing $\left[\frac{3}{2}, 3\right]$ (if $\Omega$ is $\mathscr{C}^{1}$, then this interval is $(1, \infty))$. This led M. Taylor in [50] to formulate the conjecture that the Dirichlet-Stokes semigroup defined originally on $H_{D}$ extends to an analytic semigroup on $L^{p}$ for $p$ in the same interval as in [19]. This is actually true as shown in Subsection 1.1.2. It is not known whether this range is optimal, i.e., for any $p>3$ (or any $p<\frac{3}{2}$ ), is there a bounded Lipschitz domain such that the Dirichlet-Stokes semigroup $\left(e^{-t A_{D}}\right)_{t \geq 0}$ does not extend to a bounded analytic semigroup in $H_{D}^{p}$ ? When considering Hodge boundary conditions ( $\mathrm{Hbc}$ ), the range where $\left(e^{-t A_{T}}\right)_{t \geq 0}$ extends to a bounded analytic semigroup in $H_{D}^{p}$ is however larger (see Remark 3.12, based on results in [29]).

To apply the Fujita-Kato scheme as in Subsection 1.2, proving that the Dirichlet-Stokes semigroup $\left(e^{-t A_{D}}\right)_{t \geq 0}$ extends to an analytic semigroup in $H_{D}^{3}$ seems to be the first step to obtain mild solutions of the Navier-Stokes system with Dirichlet boundary conditions. Next step is to be able to estimate $\nabla e^{-t A_{D}}$ in the $L^{3}$ norm, which is not as straightforward as in the $L^{2}$ case where $\left\|\nabla e^{-t A_{D}} f\right\|_{2}=\left\|A_{D}^{1 / 2} e^{-t A_{D}} f\right\|_{2}$.

Finally, it would be very satisfactory to obtain a theory for Robin boundary conditions (Rbc) in Lipschitz domains as studied in Section 4 for $\mathscr{C}^{1}$ domains.

\section{References}

[1] T. Akiyama, H. Kasai, Y. Shibata, and M. Tsutsumi, On a resolvent estimate of a system of Laplace operators with perfect wall condition, Funkcial. Ekvac. 47 (2004), no. 3, 361-394. 3

[2] Herbert Amann, On the strong solvability of the Navier-Stokes equations, J. Math. Fluid Mech. 2 (2000), no. 1, 16-98. 1.2

[3] C. Amrouche, C. Bernardi, M. Dauge, and V. Girault, Vector potentials in threedimensional non-smooth domains, Math. Methods Appl. Sci. 21 (1998), no. 9, 823-864. 3.2

[4] C. Bègue, C. Conca, F. Murat, and O. Pironneau, Les équations de Stokes et de NavierStokes avec des conditions aux limites sur la pression, Nonlinear partial differential equations and their applications. Collège de France Seminar, Vol. IX (Paris, 1985-1986), Pitman Res. Notes Math. Ser., vol. 181, Longman Sci. Tech., Harlow, 1988, pp. 179-264. 3, (document)

[5] H. Beirão da Veiga, Remarks on the Navier-Stokes evolution equations under slip type boundary conditions with linear friction, Port. Math. (N.S.) 64 (2007), no. 4, 377-387. 4

[6] Russell M. Brown, The method of layer potentials for the heat equation in Lipschitz cylinders, Amer. J. Math. 111 (1989), 339-379. 1.1.2 
[7] Russell M. Brown and Zhongwei Shen, Estimates for the Stokes operator in Lipschitz domains, Indiana Univ. Math. J. 44 (1995), no. 4, 1183-1206. 1.1.1

[8] L. Caffarelli and I. Peral, On $W^{1, p}$ estimates for elliptic equations in divergence form, Commun. Pure Appl. Math. 51 (1998), 1-21. 1.1.2

[9] Martin Costabel, A remark on the regularity of solutions of Maxwell's equations on Lipschitz domains, Math. Methods Appl. Sci. 12 (1990), no. 4, 365-368. 3.2

[10] Martin Costabel and Alan McIntosh, On Bogovskiน and regularized Poincaré integral operators for de Rham complexes on Lipschitz domains, Math. Z. 265 (2010), no. 2, 297-320. 3.1

[11] Bjorn Dahlberg, Carlos Kenig, and Gregory Verchota, Boundary value problems for the system of elastostatics in Lipschitz domains, Duke Math. J. 57 (1988), 795-818. 1.1.2

[12] Georges de Rham, Differentiable manifolds, Grundlehren der Mathematischen Wissenschaften [Fundamental Principles of Mathematical Sciences], vol. 266, SpringerVerlag, Berlin, 1984, Forms, currents, harmonic forms, Translated from the French by F. R. Smith, With an introduction by S. S. Chern. 1.1.1

[13] Luciano de Simon, Un'applicazione della teoria degli integrali singolari allo studio delle equazioni differenziali lineari astratte del primo ordine, Rend. Sem. Mat. Univ. Padova 34 (1964), 205-223. 1.2

[14] Paul Deuring, The Stokes resolvent in 3D domains with conical boundary points: nonregularity in $L^{p}$-spaces, Adv. Differential Equations 6 (2001), no. 2, 175-228. 1.1.2, 3.13, 4.3

[15] Paul Deuring and Wolf von Wahl, Strong solutions of the Navier-Stokes system in Lipschitz bounded domains, Math. Nachr. 171 (1995), 111-148. 1

[16] Martin Dindoš and Marius Mitrea, The stationary Navier-Stokes system in nonsmooth manifolds: the Poisson problem in Lipschitz and $C^{1}$ domains, Arch. Ration. Mech. Anal. 174 (2004), no. 1, 1-47. 1.1.1

[17] L. Roland Duduchava, Dorina Mitrea, and Marius Mitrea, Differential operators and boundary value problems on hypersurfaces, Math. Nachr. 279 (2006), no. 9-10, 996-1023. (document)

[18] Eugene Fabes, Carlos Kenig, and Gregory Verchota, The Dirichlet problem for the Stokes system on Lipschitz domains, Duke Math. J. 57 (1988), 769-793. 1.1.2

[19] Eugene Fabes, Osvaldo Mendez, and Marius Mitrea, Boundary layers on Sobolev-Besov spaces and Poisson's equation for the Laplacian in Lipschitz domains, J. Funct. Anal. 159 (1998), no. 2, 323-368. 3.1, 4.2, 4.3

[20] Hiroshi Fujita and Tosio Kato, On the Navier-Stokes initial value problem. I, Arch. Rational Mech. Anal. 16 (1964), 269-315. (document), 1, 1.2

[21] Yoshikazu Giga, Analyticity of the semigroup generated by the Stokes operator in $L_{r}$ spaces, Math. Z. 178 (1981), no. 3, 297-329. 1.1.2, 4.3 
[22] Yoshikazu Giga and Tetsuro Miyakawa, Solutions in $L_{r}$ of the Navier-Stokes initial value problem, Arch. Rational Mech. Anal. 89 (1985), no. 3, 267-281. 4.3

[23] V. Girault, Curl-conforming finite element methods for Navier-Stokes equations with nonstandard boundary conditions in $\mathbf{R}^{3}$, The Navier-Stokes equations (Oberwolfach, 1988), Lecture Notes in Math., vol. 1431, Springer, Berlin, 1990, pp. 201-218. 3, (document)

[24] T. Iwaniec and C. A. Nolder, Hardy-Littlewood inequality for quasiregular mappings in certain domains in $\mathbf{R}^{n}$, Ann. Acad. Sci. Fenn. Ser. A I Math. 10 (1985), 267-282. 1.1.2

[25] David Jerison and Carlos E. Kenig, The inhomogeneous Dirichlet problem in Lipschitz domains, J. Funct. Anal. 130 (1995), no. 1, 161-219. 1.1.1

[26] Carlos E. Kenig, Harmonic analysis techniques for second order elliptic boundary value problems, CBMS Regional Conference Series in Mathematics, vol. 83, Published for the Conference Board of the Mathematical Sciences, Washington, DC; by the American Mathematical Society, Providence, RI, 1994. 1.1.2

[27] P. G. Lemarié-Rieusset, Recent developments in the Navier-Stokes problem, Chapman \& Hall/CRC Research Notes in Mathematics, vol. 431, Chapman \& Hall/CRC, Boca Raton, FL, 2002. 1.2

[28] Jacques-Louis Lions, Espaces d'interpolation et domaines de puissances fractionnaires d'opérateurs, J. Math. Soc. Japan 14 (1962), 233-241. 1.1.1, 2.1

[29] Alan McIntosh and Sylvie Monniaux, Hodge-Dirac, Hodge-Laplacian and Hodge-Stokes operators in $L^{p}$ spaces on Lipschitz domains, In preparation, 35 pages, 2015. 3, 3.1, 3.1, $3.1,3.12,4.3$

[30] Dorina Mitrea, Marius Mitrea, and Sylvie Monniaux, The Poisson problem for the exterior derivative operator with Dirichlet boundary condition in nonsmooth domains, Commun. Pure Appl. Anal. 7 (2008), no. 6, 1295-1333. 3.1

[31] Dorina Mitrea, Marius Mitrea, and Michael Taylor, Layer potentials, the Hodge Laplacian, and global boundary problems in nonsmooth Riemannian manifolds, Mem. Amer. Math. Soc. 150 (2001), no. 713, x+120. 3.2, 3.3, 4.2

[32] Marius Mitrea, Sharp Hodge decompositions, Maxwell's equations, and vector Poisson problems on nonsmooth, three-dimensional Riemannian manifolds, Duke Math. J. 125 (2004), no. 3, 467-547. 4.1, 4.1, 4.1, 4.1

[33] Marius Mitrea and Sylvie Monniaux, The regularity of the Stokes operator and the Fujita-Kato approach to the Navier-Stokes initial value problem in Lipschitz domains, J. Funct. Anal. 254 (2008), no. 6, 1522-1574. 1, 1.1 .1

[34] Marius Mitrea and Sylvie Monniaux, The nonlinear Hodge-Navier-Stokes equations in Lipschitz domains, Differential Integral Equations 22 (2009), no. 3-4, 339-356. 3, (document), 3, 3.1, 3.2

[35] Marius Mitrea and Sylvie Monniaux, On the analyticity of the semigroup generated by the Stokes operator with Neumann-type boundary conditions on Lipschitz subdomains of Riemannian manifolds, Trans. Amer. Math. Soc. 361 (2009), no. 6, 3125-3157. 3, 3.1 
[36] Marius Mitrea, Sylvie Monniaux, and Matthew Wright, The Stokes operator with Neumann boundary conditions in Lipschitz domains, J. Math. Sci. (N. Y.) 176 (2011), no. 3, 409-457. 2, 2.1, 2.4, 2.1, 2.2, 2.2

[37] Sylvie Monniaux, Uniqueness of mild solutions of the Navier-Stokes equation and maximal $L^{p}$-regularity, C. R. Acad. Sci. Paris Sér. I Math. 328 (1999), no. 8, 663-668. 1.2, 1.2

[38] Sylvie Monniaux, On uniqueness for the Navier-Stokes system in 3D-bounded Lipschitz domains, J. Funct. Anal. 195 (2002), no. 1, 1-11. 1.2

[39] Sylvie Monniaux, Navier-Stokes equations in arbitrary domains: the Fujita-Kato scheme, Math. Res. Lett. 13 (2006), no. 2-3, 455-461. 1

[40] Sylvie Monniaux, Maximal regularity and applications to PDEs, Analytical and numerical aspects of partial differential equations, Walter de Gruyter, Berlin, 2009, pp. 247-287. 1.2

[41] Sylvie Monniaux and El Maati Ouhabaz, The Incompressible Navier-Stokes System with Time-Dependent Robin-Type Boundary Conditions, J. Math. Fluid Mech. 17 (2015), no. 4, 707-722. 3.1, 4, 4.1

[42] Sylvie Monniaux and Jürgen Saal, Robin-Hodge boundary conditions for the NavierStokes equations in 3D-bounded Lipschitz domains, In preparation, 2015. 4

[43] Barrett O'Neill, Elementary differential geometry, second ed., Elsevier/Academic Press, Amsterdam, 2006. (document)

[44] Zhongwei Shen, Boundary value problems for parabolic Lamé systems and a nonstationary linearized system of Navier-Stokes equations in Lipschitz cylinders, Amer. J. Math. 113 (1991), 293-373. 1.1.2, 1.2

[45] Zhongwei Shen, Resolvent estimates in $L^{p}$ for elliptic systems in Lipschitz domains, J. Funct. Anal. 133 (1995), 224-251. 1.1.2

[46] Zhongwei Shen, Bounds of Riesz transforms on $L^{p}$ spaces for second order elliptic operators, Ann. Inst. Fourier (Grenoble) 55 (2005), 173-197. 1.1.2

[47] Zhongwei Shen, Necessary and sufficient conditions for the solvability of the L ${ }^{p}$ Dirichlet problems on Lipschitz domains, Math. Ann. 336 (2006), 697-724. 1.1.2

[48] Zhongwei Shen, Resolvent estimates in $L^{p}$ for the Stokes operator in Lipschitz domains, Arch. Ration. Mech. Anal. 205 (2012), no. 2, 395-424. 1, 1.1.2, 1.1.2, 1.1.2, 1.1.2, 1.1.2, $1.1 .2,3.13$

[49] Michael E. Taylor, Partial differential equations. I, Applied Mathematical Sciences, vol. 115, Springer-Verlag, New York, 1996, Basic theory. 3

[50] Michael E. Taylor, Incompressible fluid flows on rough domains, Semigroups of operators: theory and applications (Newport Beach, CA, 1998), Progr. Nonlinear Differential Equations Appl., vol. 42, Birkhäuser, Basel, 2000, pp. 320-334. 1.1.2, 3.13, 4.3 
[51] Roger Temam, Navier-Stokes equations, revised ed., Studies in Mathematics and its Applications, vol. 2, North-Holland Publishing Co., Amsterdam-New York, 1979, Theory and numerical analysis, With an appendix by F. Thomasset. 1.1.1

[52] Gregory Verchota, Layer potentials and regularity for the Dirichlet problem for Laplace's equation in Lipschitz domains, J. Funct. Anal. 59 (1984), 572-611. 1.1.2 